\title{
Spectroelectrochemistry of Quantum Dots
}

\author{
Jesus Garoz-Ruiz, ${ }^{[\mathrm{a}]}$ Juan V. Perales-Rondon, ${ }^{[\mathrm{a}]}$ Aranzazu Heras, ${ }^{[\mathrm{a}]}$ and Alvaro Colina* ${ }^{[\mathrm{a}]}$ \\ Dedication((optional))
}

\begin{abstract}
Spectroelectrochemistry (SEC) is a set of techniques with many advantages in the study and characterization of materials. Although SEC has not yet been widely used to study quantum dots (QDs), the information extracted from SEC experiments about these nanostructures is very useful. Most of the works that use SEC to study QDs are high-quality pieces of research. This review intends to show how to perform SEC in an easy way and what information can be obtained using these techniques.
\end{abstract}

Most of the examples shown in this review are related to semiconductor and carbon QDs. After a brief introduction, some optoelectronic properties of QDs and the main SEC techniques are described. The capabilities of SEC for the study of QDs are illustrated with examples extracted from literature. Finally, the needs of SEC to become a user-friendly technique and its evolution to become more powerful are commented in the last section of the review.

Keywords: Spectroelectrochemistry • Quantum Dots $\bullet U V / V I S / N I R \cdot P h o t o l u m i n e s c e n c e \cdot R a m a n$

\section{Introduction}

Since the end of the $20^{\text {th }}$ century, nanotechnology is opening a number of exciting possibilities in almost all fields of science. The number of works about nanostructures is growing year by year, but, in general, there is still no clear definition or classification for all the existing nanostructures. For example, the term quantum dot (QD) is applied to a few-nanometre material that shows quantum confinement which provides very interesting properties to these nanoentities. A number of diverse QDs with different composition have been shown in literature. ${ }^{[1-12]}$ Most of them are nanocrystals (NCs) with semiconductor properties, but also this terminology has been applied to carbon nanostructures. All the nanostructures included in the term QD have in common the nanometre size of the structure, which usually provides a quantum confinement effect that originates a particular density of electronic states, inducing the energy level separation ${ }^{[2,13-15]}$ and producing an increase of the bandgap (HOMO-LUMO gap, $\mathrm{E}_{\mathrm{g}}$ ) with decreasing size. The appearance of discrete energy levels near the band edges implies very interesting optoelectronic properties for QDs that strongly depend on their size- and shape. Obviously, density of electronic states depends on the composition and the type of atoms of these nanoparticles (NPs), with the properties being very different depending on its nature (semiconductor nanocrystals, carbon nanostructures, etc.).

The range of fundamental properties of a QD with a single chemical composition is enormous depending on the size and shape of the nanostructure. Furthermore, by tailoring the chemical composition, the properties can be completely changed. Therefore, a new world of possibilities in chemistry of materials appeared with the synthesis of these amazing nanomaterials. Among all features of QDs, optoelectronic properties are perhaps the most interesting ones for a number of applications. Therefore, suitable tools to study this type of properties are needed. Characterization at the nanoscale is fundamental to know what nanostructure is being studied, but instrumental techniques at the macroscale are also essential not only to understand the properties of these nanomaterials but also to design materials for specific purposes.

In this sense, spectroelectrochemistry (SEC) should be very useful to study the optoelectronic properties of QDs since this set of techniques combine optical (spectral) and electrochemical information.

Spectroelectrochemistry can be defined as a set of instrumental techniques that combine an optical spectroscopy with an electrochemical technique. Depending on the combinations, very different SEC techniques can be defined. The most used SEC technique is Ultraviolet/Visible (UV/VIS) absorption SEC (UV/VIS-SEC) which combines UV/VIS absorption spectroscopy with an electrochemical technique, usually voltammetry or chronoamperometry. Depending on the chemical system under study, many other combinations can be used. The spectral range can be extended to the infrared (IR), obtaining IR absorption SEC and Near-IR absorption SEC (NIR-SEC). Photoluminescence SEC (PL-SEC) often combines fluorescence spectroscopy with voltammetry or chronoamperometry. In the same way, Raman-SEC combines Raman spectroscopy with voltammetry or

[a] Department of Chemistry, Universidad de Burgos Pza. Misael Bañuelos s/n, E-09001 Burgos, Spain phone: +34947258817 e-mail: acolina@ubu.es 
chronoamperometry. Electrochemistry (EC) has also been combined with electron paramagnetic resonance (EPR) and nuclear magnetic resonance (NMR). Therefore, the nature of the information provided by SEC depends on the optical and electrochemical techniques selected.

The capabilities of SEC for characterization of materials have been largely demonstrated along the years. However, this set of techniques has not been widely used for characterization of QDs, probably because up to now it was not easy to perform SEC experiments. SEC instruments were typically designed and constructed by users, combining two independent instruments in customized setups. Additionally, SEC cells were quite complicated to use and, sometimes, they presented low reproducibility. Nowadays, commercial instruments are available and some of them include software not only to register concomitantly the two types of signals, but also to analyse the huge amount of data obtained in each experiment.

This review is limited to the use of SEC for studying QDs, without discussion about electrochemiluminescence (ECL) or photo-electrochemistry (PEC), although SEC instruments can also be useful to perform ECL and PEC experiments.

\section{Quantum Dots Structure and Properties}

The term QD has been applied to different nanostructures, but the most usual meaning is referred to semiconductor QDs. These QDs are zero-dimensional NCs with respect to the bulk material. ${ }^{[7]}$ QDs have even been described as artificial atoms. ${ }^{[4,6]}$ The properties of semiconductor QDs emerge not only from their composition but also from their small size, which yields a large surface-to-volume ratio and a quantum confinement behaviour. Their properties also depend on the existence of surface and trap states, the presence of impurities and the existence of different nature capping layers. ${ }^{[14,16]}$ Compared to single component nanostructures, the properties of hybrid nanomaterials can be modified by varying the composition of their chemical components, and related parameters such as morphology and capping layers. ${ }^{[17]}$

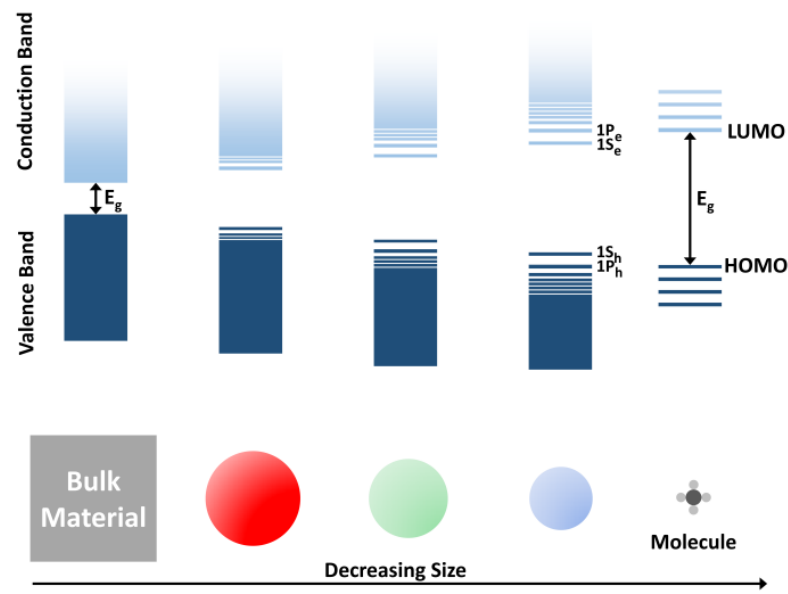

Figure 1. Schematic representation of the energy level structure for a semiconductor bulk material, semiconductor QDs and a molecule.
Alvaro Colina received his B.S. in Chemistry from Universidad de Valladolid and his Ph.D. in Chemistry from Universidad de Burgos, Spain, where he is working as associated professor. His research interests are devoted to the development of new multi-response instrumental techniques. Particularly, he has developed a number of different SEC techniques and devices that have been applied in the characterization of materials, study of reaction mechanisms and for analytical purposes.

Aranzazu Heras received her BS in Chemistry and her PhD in Chemistry from Universidad de Burgos (Spain). She is associate professor in Analytical Chemistry from 1997. Her research interests include different aspects related to SEC (UV-Vis-NIR, photoluminescence, Raman), from design and development of new devices to applications of this technique in analysis, characterization of organic, inorganic and nanomaterials, and in the study of different reaction mechanisms.

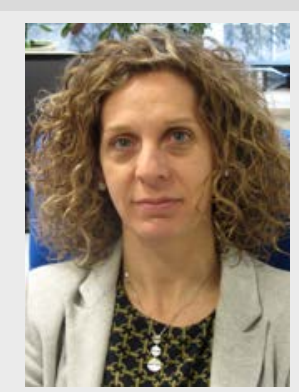

Juan V. Perales-Rondon received his B.S. in Chemistry from Universidad de Los Andes (Venezuela) and his Ph.D. in Electrochemistry from the Universidad de Alicante (Spain), working in electrocatalysis, in understanding the mechanism of formic acid oxidation on $\mathrm{Pt}$ electrodes. Then he moved to the Universidad de Burgos to work in the SEC field and the development of new SEC devices. Recently he is working in the use of Raman-SEC to develop different analytical applications.

Jesus Garoz-Ruiz received his B.S. and Ph.D. in Chemistry from Universidad de Burgos. Since 2017, he is working as postdoctoral researcher. $\mathrm{He}$ has developed different devices based on optical fibers coupled to optically transparent electrodes made of nanomaterials and to screen-printed electrodes. His research is mainly focused on the use of the novel devices to study biomolecules and to demonstrate the advantages of SEC, particularly in the field of quantitative analysis.

Metals and semiconductors exhibit extended band structures due to the overlapping of atomic orbitals. The density of states (DOS) within a band depends on the number of atoms of an ensemble. The evolution of the DOS with material size is shown in Figure 1. QDs lie in between the atomic and molecular limit of discrete DOS and the extended crystalline limit of continuous bands. Thus, there is a size for any material below which there is a substantial variation of fundamental optoelectronic properties with size. ${ }^{[13,18]}$ At sizes smaller than the exciton Bohr radius, quantum effects lead to an increased bandgap and quantized energy levels. ${ }^{[19]}$ 
One of the most remarkable properties of QDs is the dependence of their electronic structure with size. The smaller the QDs, the higher the energy shifting of the electronic excitations, and the oscillator strength is concentrated into just a few transitions. ${ }^{[5]}$ Quantum confinement effect implies an increase of the band-gap energy (blue shifting) when the QD diameter decreases. $^{[6,20]}$ Obviously, this effect depends on the type of semiconductor.

Optical absorption spectroscopy is a widely used technique to study the quantum effects in QDs. By using this technique, the development of discrete features in the spectra and the enlargement of the energy gap in semiconductor QDs can be observed. ${ }^{[5]}$ For example, empirical relationships between the size and the first absorption wavelength have been used to calculate and calibrate the size and size-distribution of different QDs. ${ }^{[21]}$ These empirical relationships were obtained for different QDs. In the case of CdTe QDs, the diameter can be calculated as follows, eq. 1 :

$$
\begin{aligned}
& \mathrm{D}=\left(9.8127 \times 10^{-7}\right) \lambda^{3}-\left(1.7147 \times 10^{-3}\right) \lambda^{2}+ \\
& (1.0064) \lambda-194.84
\end{aligned}
$$

where $\mathrm{D}(\mathrm{nm})$ is the diameter and $\lambda$ is the first excitonic absorption peak of the CdTe QDs, respectively.

PL spectroscopy can be considered as one of the most useful techniques to explore the optical properties of QDs, due to the characteristic luminescence of these nanomaterials. This technique helps to remove the size distribution broadening effect on the absorption spectrum of QDs, as well as in assigning the discrete optical transitions. ${ }^{[5]}$

After the excitation of QDs by photons, electron and hole possess high energies because of the transition of the electron from the ground state to an excited one. The energies associated with these optical absorptions are determined by the electronic structure of the material. The excited electron and the hole may form an exciton. The electron may recombine with the hole and relax to a lower energy state, ultimately reaching the ground state. The excess energy resulting from recombination and relaxation may be either radiative (emission of photons) or non-radiative (emission of phonons or Auger electrons). ${ }^{[7,22]}$ Radiative events can be: (i) band-edge emissions, due to the recombination of an excited electron in the conduction band with a hole in the valence band; (ii) defect emissions that come from localized impurities and/or trap states in the band-gap and (iii) emissions that take place when impurities are intentionally incorporated to provoke an extrinsic luminescence. ${ }^{[7,22]}$ It is noteworthy that absorption of photons by QDs may not result in emission of light. Electrons and holes in excited states may also return to lower energy and ground states by non-radiative relaxations that can be classified in internal and external conversion. Deep level traps have a tendency to undergo non-radiative recombination by emitting phonons. ${ }^{[7]}$ Surface defects are dominant channels for non-radiative decay of carriers.

Förster (or fluorescence) resonance energy transfer (FRET) is a non-radiative energy transfer process from a fluorescent donor to a lower energy acceptor via longrange dipole-dipole interactions. FRET-based technologies play a significant role in biosensing, with
QDs providing potential solutions to problems such as photobleaching or significant emission overlap between donor and acceptor. ${ }^{[23,24]}$ FRET can be detected by monitoring the acceptor fluorescence and/or the acceptor quenching.

Some photophysical properties of QDs can be disadvantageous. For example, blinking is a property of QDs referred to a random alternation between an emitting state and a non-emitting state. In any case, QD blinking can be suppressed in different ways. ${ }^{24]}$

The particular electronic structure of QDs confers very interesting properties to these nanomaterials ${ }^{[20,23]}$ such as higher extinction coefficients and quantum yields (QYs). Usually, QDs exhibit broad excitation bands but narrow emission peaks, long fluorescence lifetimes, a bright and photostable emission and a largeeffective Stokes shift. As has been stated above, absorption and emission can be tuned with size and composition, obtaining emissions from the UV to the NIR spectral regions. Moreover, a significant advance in the functionalization of QDs has been carried out in the last years. Optical properties of QDs can be modified using, for example, surface ligands that provoke the shifting of the emission or absorption bands, or the enhancement or quenching of the PL, allowing to modulate the PL for different applications.

Electronic doping is one of the most important experimental capabilities in semiconductor research and technology. ${ }^{[25]}$
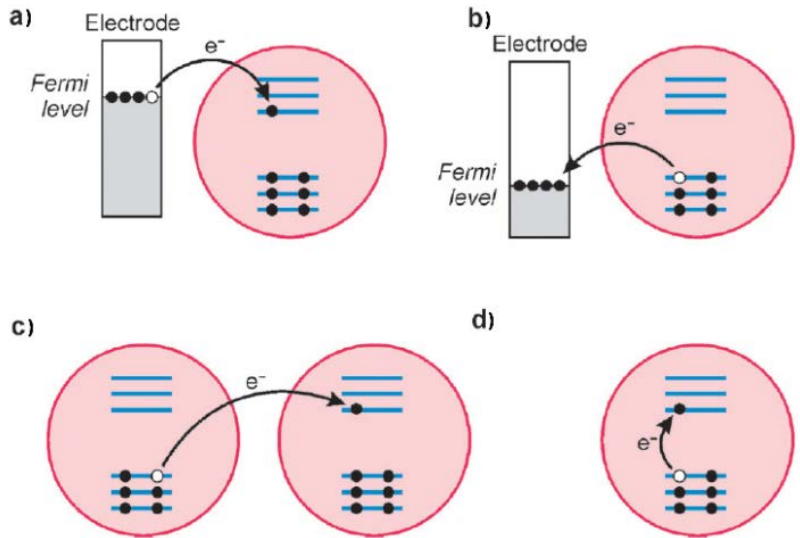

d)

Figure 2. Schematic representation of charge-transfer processes involving semiconductor nanoparticles: (a) electron transfer from a metal electrode to a neutral particle; (b) electron extraction from the particle by a metal electrode (hole injection); (c) simultaneous injection of an electron and a hole in two non-interacting particles; (d) generation of an electronhole pair within the same particle. Reprinted with permission from Ref. [26] Copyright 2012 Royal Society of Chemistry..

Four different types of charge-transfer processes have been proposed for QDs, [26,27] which are summarized in Figure 2. (a) electron injection into a neutral nanoparticle, Figure 2.a, (b) hole injection or electron extraction from a neutral nanoparticle, Figure 2.b, (c) simultaneous injection of an electron and a hole in two non-interacting QDs, which ideally corresponds to withdrawing an electron from one particle and placing it into an identical particle at infinite distance, Figure 2.c, and (d) generation of an electron-hole pair 
within the same particle caused by optical excitation, Figure 2.d.

Electrochemistry can be used to inject electrons and holes from an electrode into the quantum confined states of a semiconductor. Electrochemistry of QDs can provide information on the absolute energies of the valence and conduction bands (from the redox potential values) and on the rate constants of the heterogeneous electron-transfer processes involving the nanocrystal. ${ }^{[26,28]}$

Electrochemical measurements of QDs in solution are difficult to carry out due to the low solubility and the small diffusion coefficients of these NPs. Therefore, poor signal-to-noise $(\mathrm{S} / \mathrm{N})$ ratios are obtained, with the intensities related to the QDs being difficult to be measured with respect to the background signal. ${ }^{[28]}$ For this reason, in some cases SEC measurements can help in the study of the redox processes and in the determination of the corresponding potential values. In addition, QD samples adsorbed on the working electrode have turned out to be much more useful to obtain the electrochemical information. ${ }^{[29]}$

The electrochemical bandgap energy $\Delta \mathrm{E}_{\mathrm{el}}$, calculated from the difference between the potentials at which oxidation and reduction of the QDs take place, can be related to the optical bandgap energy $\Delta \mathrm{E}_{\mathrm{op}}$, determined from spectroscopy, by eq. 2: ${ }^{[28]}$

$\Delta E_{\text {op }}=\Delta E_{e l}-J_{e-h}$

in which $J_{e-h}$ is the total Coulomb energy of the electronhole pair. Hence, $\Delta E e l$ is expected to be larger than $\Delta E_{\text {op }}{ }^{[28]}$ for any given QD.

SEC should provide all this information in a single experiment, and what is much more important, exactly about the same sample. The two measurements, optical and electrochemical, are obtained concomitantly on the same sample and under the same experimental conditions. It should be noted that EC measurements mainly probe the surface of the QDs, whereas spectroscopy measurements are usually related to processes that take place inside the NPs. ${ }^{[26]}$

All these optoelectronic properties make QDs very useful for a number of applications in very different fields such as electroluminescent, photovoltaics, optoelectronic and electrochromic devices, light sources, light converters, photodetectors, bio-sensing and bioimaging, among others. ${ }^{[11,12,30-34]}$

With respect to carbon QDs those related with graphene are highly interesting. The UV/VIS absorption spectrum of graphene QDs (GQDs) shows two main peaks, one around $230 \mathrm{~nm}$ assigned to $\pi-\pi^{*}$ transition of $\mathrm{C} \mathrm{sp}{ }^{2}$ bonds and a shoulder at about $300 \mathrm{~nm}$ related to $\mathrm{n}-\pi^{*}$ transitions of $\mathrm{C}=\mathrm{O}$ or $\mathrm{C}-\mathrm{O}$ bonds. ${ }^{[1,35-37]}$ As in the case of QDs, GQDs show PL at very different wavelengths depending on its size, from deep ultraviolet to red. ${ }^{[36]}$ The PL mechanism is related to the quantum confinement effect, emissive traps, excitons of carbon, aromatic structures, oxygen-containing groups, free zigzag sites and edge defects. PL depends not only on size, but also on the excitation wavelength, oxidation degree, solvent, $\mathrm{pH}$, functionalization, etc. ${ }^{1,35-37]}$

A number of potential applications have been proposed for GQDs. ${ }^{[8,38]}$ The most attractive characteristic of GQDs with respect to semiconductor
QDs is the high availability of carbon. Moreover, in principle, GQDs show very low toxicity, ${ }^{[3]}$ therefore high biocompatibility, and they can be solubilized in very different solvents. The possibility of the functionalization of their edges is also very important for a number of applications. ${ }^{[8]}$ However, this new nanomaterial needs to be studied in a deeper way.

\section{Spectroelectrochemistry}

As can be deduced from the previous section, QDs are one of the most important luminescent materials, representing a revolution in the design of luminescent systems for very different applications. SEC is particularly useful to study QDs because of their unique optical properties that can be tuned using electrochemistry. Thus, QDs exhibit several advantages in SEC, such as characteristic energy gap and luminescence with high extinction coefficients and QYs, size-tunable spectrum, high optical stability, easy modification of optical properties, controlled nonradiative processes and electronic doping, among others. In this section, we will describe different SEC techniques that have been used to study and characterize QDs.

\subsection{UVIVIS/NIR Absorption Spectroelectrochemistry}

The combination of EC and UV/VIS/NIR absorption spectroscopy is probably the most reported SEC technique in the literature. ${ }^{[39-41]}$ This section is focused on how to measure using UV/VIS/NIR-SEC. The main instrumentation required to perform a UV/VIS/NIR absorption SEC experiment consists of a potentiostat, a light source, a spectroelectrochemical cell and a spectrometer, Figure 3. Besides, a trigger is required to ensure the correct synchronization between potentiostat and spectrometer. The light beam can be conducted between the different parts using lenses, mirrors and/or optical fibers. ${ }^{[39-41]}$

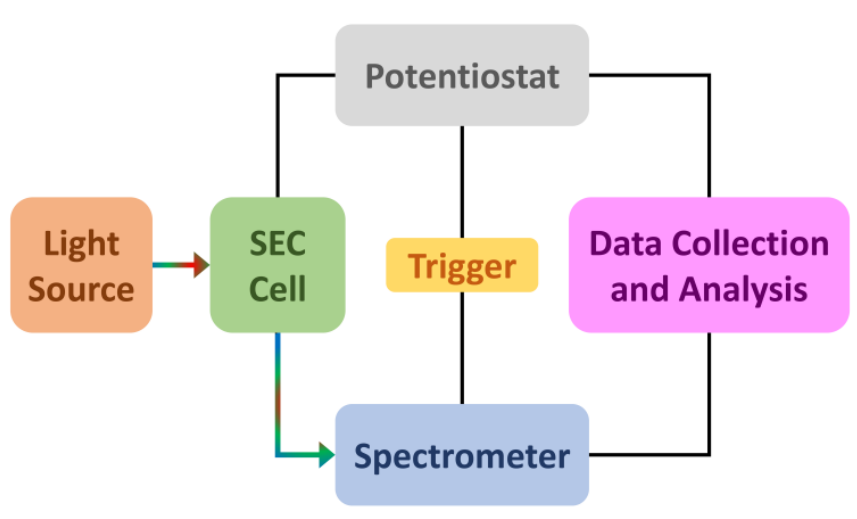

Figure 3. Typical components of a SEC instrument.

The potentiostat controls the redox state of the sample, and at the same time, it records the electrochemical signal during the experiment. With respect to the SEC cell, a three-electrode system is usually employed to control the potential during the 
experiment. Working electrode (WE), reference electrode (RE) and counter-electrode (CE) must be placed in a suitable position in the SEC cell to obtain high-quality electrochemical signals and to avoid interferences in the optical path. The light source provides the electromagnetic radiation that interrogates the sample from an optical point of view during the electrochemical process. The choice of a good light source for the studied spectral region is mandatory.

Optical setups can be quite complex because it is not easy to conduct the light beam from the light source to the sample and from the sample to the spectrometer. Optical fibers have undoubtedly helped to simplify optical setups, ${ }^{[42,43]}$ allowing us to work with few microliters of solution. Light can be conducted and collected in close proximity to the electrode surface in a much simpler way using optical fibers than with an optical bench.

The key point in a SEC experiment is the SEC cell, which defines the type of UV/VIS/NIR-SEC experiment to be performed (see section 3.1.1).

Finally, the spectrometer is mainly composed of a monochromator and a detector. The monochromator defines the spectral range and the resolution of the experiment. Scanning monochromators as dispersing elements are rarely used in SEC because, as the spectra evolve with potential/time, it is not recommended to scan the wavelengths during the experiment. Diode array detectors are more useful for SEC than photomultiplier tubes because, although the latter can be more sensitive than a diode array detector, they allow to measure only one wavelength in time-resolved SEC experiments, thus losing the information about the evolution of full spectra that is obtained with a diode array detector. The integration time for the data acquisition is one of the key parameters for SEC measurements because the lower the integration time, the higher the optical information about the electrochemical process. In fact, the real power of SEC is reached when spectroscopy is used in a similar dynamic way to EC, recording both the electrochemical and the optical responses in a continuous and fast way during the whole SEC experiment

\subsubsection{UVIVIS/NIR-SEC Optical Configurations and Cells.}

Very different SEC devices have been developed and fabricated to solve specific problems. ${ }^{[39-41]}$ Given the impossibility of referencing all of them, the main configurations in which they can be grouped are explained in Figure 4, particularly normal transmission, normal reflection, angle reflection, parallel, and attenuated total reflection (ATR) arrangements. It should be noted that SEC can be performed in a thinlayer or in a semi-infinite diffusion regime. Currently, the technical developments allow the researcher to perform SEC experiments in a much easier, quicker and reproducible way than few years ago, using volumes of only microliters ${ }^{[43]}$ and with a great variety of materials as electrodes, even screen-printed electrodes (SPEs). ${ }^{[42]}$ Nowadays, the difficulty of performing a SEC experiment is practically the same as that for an EC measurement.

In normal transmission configuration, Figure 4.a, the light beam travels perpendicularly to the electrode, crossing it and sampling both the solution and the electrode surface. ${ }^{[39-41]}$ The electrode must be electrically conductive and optically transparent in the spectral region of interest, limiting the number of electrodes that can be used. For this reason, indium tin oxide (ITO) and metallic meshes have been typically used. $^{[39-41]}$ ITO is probably the most used optically transparent electrode (OTE) to study QDs. Metallic meshes can only be used for studying processes in solution and are not easily cleaned. Thin metal films, for example gold sputtered films, ${ }^{[44]}$ constitute a good solution. Furthermore, the development of OTEs based on novel allotropes of carbon such as carbon nanotubes (CNTs) and graphene is highly promising for SEC. ${ }^{[4,45]}$ Normal transmission measurements have been usually performed in spectrophotometric cuvettes, but currently, measurements can be easily done in few-microliter solutions using optical fibers. ${ }^{\text {[4] }}$

\section{UV/VIS/NIR absorption SEC}

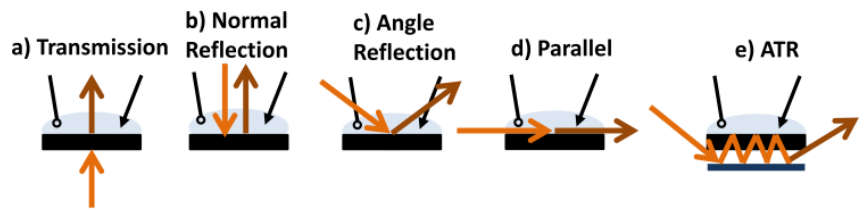

PL SEC
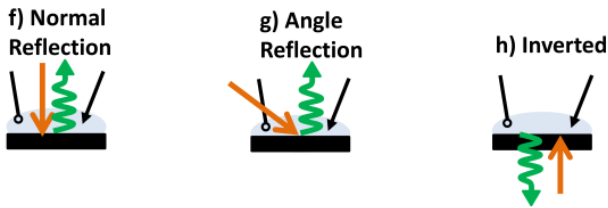

Raman SEC
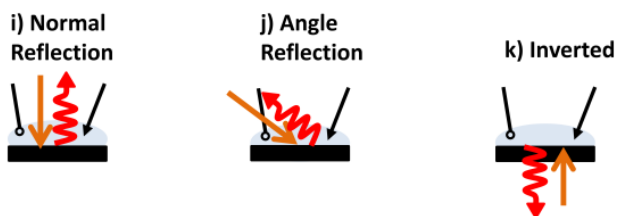

Figure 4. Typical setups for SEC. Orange arrow: incident light beam. Brown arrow: transmitted/reflected light beam. Green arrow: emitted photon. Red arrow: scattered photon. Black rectangle: WE. Black circumference: CE. Black arrow: RE

Aiming to avoid the use of OTEs and to improve the sensitivity, the normal reflection configuration was proposed, Figure 4.b. It consists of a light beam that travels perpendicularly to the electrode surface and is reflected on it, sampling the solution and the electrode surface. ${ }^{[42]}$ If the light beam is reflected on the electrode surface in a different direction than the incident light beam, the configuration is called angle reflection mode, ${ }^{[46]}$ Figure 4.c. In the reflection modes, the number of electrodes that can be used is much higher than in the normal transmission configuration, so usual electrodes made of metal or carbon and even SPEs can be used. ${ }^{[39-}$

41] It should be highlighted that the development of commercial reflection probes boosted the performance of these measurements. As a precaution, the researcher needs to be aware about the presence of films deposited 
on the electrode surface, which is a typical case in QDs, because changes of reflectivity could yield anomalous results and misleading interpretations. Nevertheless, good results are usually obtained using this configuration which can be very useful to simplify the optical setups and to select the most appropriate electrode material.

In parallel configuration, Figure 4.d, the light beam travels adjacent and parallel with respect to the electrode surface. ${ }^{[39-41]}$ In this arrangement, only the adjacent solution to the electrode is optically sampled, without obtaining information about the electrode surface. ${ }^{[43,44]}$ For compounds in solution, there is a clear increase in sensitivity because the optical path length is rather larger than the diffusion layer. However, if the compound of interest is deposited on the WE, normal configurations are the most suitable ones because they directly provide information about the electrode surface. ${ }^{[4]}$ In parallel arrangement, OTEs are not needed, increasing the number of electrodes that can be employed. ${ }^{[39-41]}$ Two of the most important drawbacks of this configuration were the difficulty to align the light beams and the complex assembly of the cells; but they have been overcome with the use of optical fibers with diameters close to the dimension of the diffusion layer. Bare optical fibers without jacketing material extraordinarily simplify the measurements in parallel configuration because they can be directly fixed to the WE without the need of a particular SEC cell, sampling the first microns of the solution adjacent to the electrode surface $^{[47]}$.

The combination of normal and parallel configuration in the same experiment is called bidimensional SEC (BSEC) and it provides information about the electrochemical processes taking place at the electrode surface and that ones in the solution adjacent to the electrode. ${ }^{[43,44]}$

Figure 5 shows a BSEC cell and illustrates how to simplify experimental setups for UV/VIS/NIR-SEC measurements ${ }^{[43]}$. The dimensions of the assembled BSEC cell are $5 \times 3.5 \times 1.6 \mathrm{~cm}$. In normal configuration, an optical fiber coupled to a collimating lens is placed at the back of the OTE, allowing the light to pass through the solution and the electrode. The transmitted light is collected by a $200 \mu \mathrm{m}$ optical fiber probe and it is conducted to the spectrometer. In parallel arrangement, a $100 \mu \mathrm{m}$ bare optical fiber is placed just in front of another one, both placed on the electrode surface, to test the solution adjacent to the electrode. The optical path length in parallel configuration is the distance between the two optical fibers. Finally, a homemade $\mathrm{Ag} / \mathrm{AgCl} / \mathrm{KCl} 3 \mathrm{M} \mathrm{RE}$ and a platinum wire $\mathrm{CE}$ are immersed in a $100 \mu \mathrm{L}$ solution drop.

As can be observed, optical fibres extremely simplify the design of SEC cells. Moreover, with their help, SEC has even been performed even inside a grapefruit $^{[47]}$.

In ATR configuration, ${ }^{[40]}$ Figure 4.e, the light beam that hits on a surface is bent normal from the surface as it passes from a higher to lower refractive index material; undergoing an internal reflection. In ATR, a high angle of incidence and a high refractive index crystal must be used. If the incident light is directed through the electrode toward the solution at an incident angle that exceeds the critical angle, total reflection of the light beam at the transparent electrode/solution interface is obtained. In cells with multiple reflections, where the evanescent field is propagated, the sensitivity in the interfacial region is significantly improved. The penetration depth of the evanescent wave depends on the excitation light, sampling strictly the interfacial region and avoiding the influence of the solvent due to the low penetration depth of the radiation into the solution, which is very important for studies in the IR region. It should be note that the WE must be transparent in ATR cells.
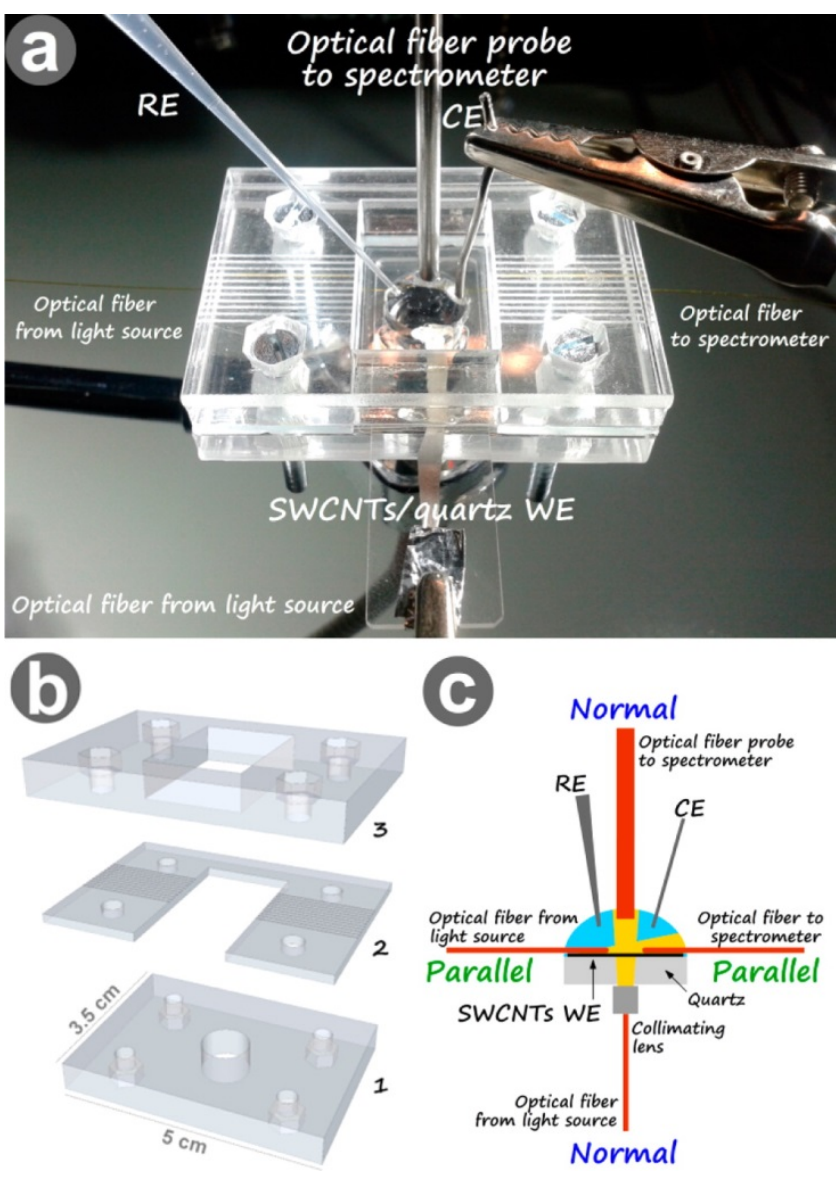

Figure 5. Bidimensional spectroelectrochemistry cell. (a) Photograph of the assembled cell ready to measure, (b) schematic view of the disassembled cell, and (c) detailed schematic view of the experimental setup. Reprinted with permission from Ref. ${ }^{[4]}$ Copyright 2015 American Chemical Society.

IR spectroelectrochemistry (IR-SEC) configurations are quite similar to the UV/VIS/NIR ones, Figure 4. Of course, light sources and spectrometers are completely different. Materials for cells and OTEs must be transparent to IR radiation and insoluble in the working solution. ${ }^{[39]}$ Solvent can absorb strongly in the IR region, therefore, configurations sampling from behind the electrode are often preferred and thin-layer cells are usually employed for studies in solution. ${ }^{[39,40]}$ Background correction for solvent IR contribution can be performed by modulation of the applied potential, polarization of the incident light, or by using a difference method as subtractively normalized interfacial Fourier transform infrared spectroscopy 
(SNIFTIRS). ${ }^{[40]}$ Electronic reflectance spectroscopy and ATR cells provide significant improvement in discrimination of background and interfacial signals. ${ }^{[40]}$ ATR configuration combined with surface-enhanced IR absorption (ATR-SEIRAS) can be used to study species deposited or adsorbed on the electrode surface. In this case, electrodes are metal films, usually of gold, vacuum evaporated or sputtered on an ATR prism. ${ }^{[39]}$

\subsection{Photoluminescence Spectroelectrochemistry}

The combination of EC and PL measurements in one experiment involves overcoming two key challenges to ensure the acquisition of high-quality PL-SEC data. The first one is related to ensuring that the excitation light beam samples the electrode-solution interface. The second challenge is related to solving instrumentally the need to collect the emitted light avoiding the interference of the incident radiation and autoabsoption processes. ${ }^{[40,48,49]}$

These two challenges have been addressed in two different ways, namely, coupling classical fluorescence setups with EC devices or coupling fluorescence microscopy with EC. As will be detailed below, each one of these couplings presents some advantages and disadvantages, being the key point the SEC cell. The main elements to perform PL-SEC experiments are similar to those described for UV/VIS/NIR-SEC, Figure 3 , with the potentiostat, the light source to excite the sample, the SEC cell with the three electrode system and the spectrometer being the main components. Light sources can be a Xenon lamp coupled with a wavelength selector, a light-emitting diode (LED) or a laser to provide a higher light intensity. LEDs and lasers help to simplify the instrumental setup. Nowadays, different wavelength LEDs are available and can be easily coupled to SEC instruments ${ }^{[50]}$, Figure 6. The detector can be either single or multi-channelled. In the first case, when only one emission wavelength is measured, a scanning monochromator can select the characteristic emission wavelength of the studied sample. However, in the second case, when a diode array detector is used, the whole emission spectrum is registered instantaneously. Finally, a trigger is required to ensure the correct synchronization between potentiostat and detector. This basic instrumental scheme suffers different modifications when fluorescence microscopy is coupled with electrochemistry mainly related to the way in which the excitation light is focalized or the emitted light is measured. As in the case of UV/VIS/NIR-SEC, optical fibers help to simplify the experimental setups. ${ }^{[51,52]}$

The link between the two types of measurements, optical and electrochemical, simultaneously performed in PL-SEC, as in other kind of SEC techniques, is the SEC cell.

\subsubsection{PL-SEC Optical Configurations and Cells}

Classical PL-SEC experiments can be performed in a very simple way using a normal reflection configuration (Figure 4.f) where the excitation light beam is conducted to the electrode interface in the same direction used to detect the emission light beam that comes from the electrode and the adjacent solution. ${ }^{[48,51,52]}$ Reflection probes, Figure 6, allow the researcher to conduct the light beam in an easy way to the electrode surface, sampling the PL that is conducted to the SEC instrument with suitable optical fibers. Different types of reflection probes are commercialy available. This zero-angle configuration allows users to carry out PL-SEC and UV/VIS/NIR-SEC. ${ }^{[52,53]}$ However, one of the most usual configurations in PL-SEC implies that the excitation and the emission light beams travels in an angle configuration (Figure 4.g), since it prevents the reflected light to reach the detector. ${ }^{[54-56]}$ Different cell designs have been proposed, some of them working with thin-layer PL-SEC cells and OTEs, ${ }^{[54,55]}$ others selecting long-optical path-length PL-SEC cells to overcome the limitation of OTEs and using metal or carbon disk electrodes. ${ }^{[56]}$ Other angles can be used in the PL-SEC cell, ${ }^{[57]}$ Figure 4.g. Moreover, if an OTE is used, light can be collected from behind the electrode surface. $^{[58,59]}$

Coupling PL-SEC with microscopy improves the sensitivity of the technique, providing significant information about the chemical systems. In epifluorescence microscopy coupled with EC is common to work with thin-layer PL-SEC cells ${ }^{[60]}$ in an inverted configuration ${ }^{[61,62]}$ (Figure 4.h), using the microscope to focus the excitation light beam on the electrode surface and measuring the emitted light beam in a zero-angle configuration thanks to a dichroic mirror and suitable filters. ${ }^{[63]}$ When confocal microscopy is coupled to PL-SEC, a pinhole is added at the confocal plane to remove the light transmitted from the sample, increasing the sensitivity by enhancing the optical resolution. ${ }^{[64,65]}$ A highly used setup combining fluorescence microscopy and electrochemistry is the socalled total internal reflection (TIRF) PL-SEC microscopy. ${ }^{[66,67]}$ The excitation light beam samples the OTE interface with an inverted configuration at an incident angle greater than the critical angle to achieve the total internal reflection of this excitation light. The generated evanescent wave excites the sample, obtaining a high sensitivity and resolution in a normal direction with respect to the electrode. The main drawback of several of these microscopy setups is the mandatory use of OTEs.

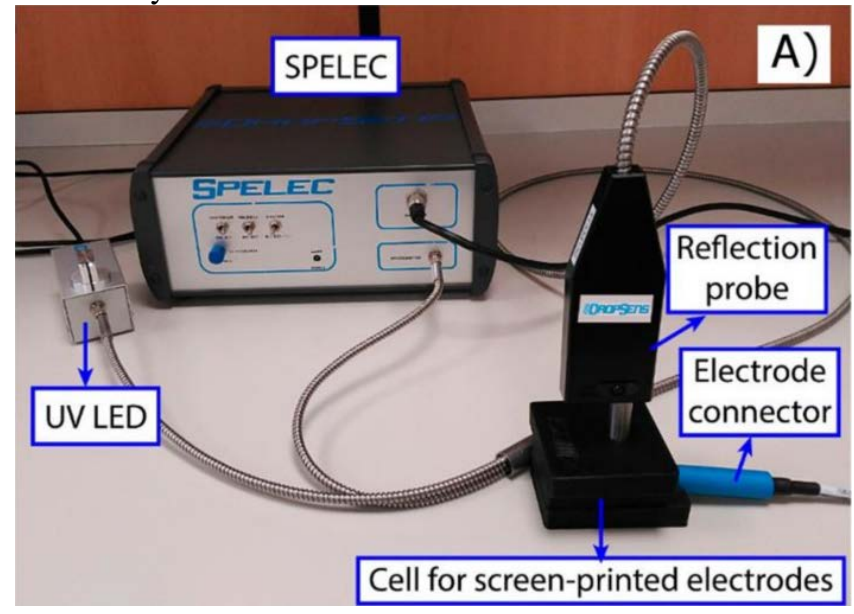

Figure 6. Image of the instrumentation employed for luminescence spectroelectrochemistry at screen-printed electrodes. Reprinted with permission from Ref. ${ }^{[50]}$ Copyright 2017 American Chemical Society. 


\subsection{Raman Spectroelectrochemistry}

Raman spectroscopy is a powerful technique to identify compounds and to study materials because it provides a real spectral fingerprint of compounds. ${ }^{[68]}$ Due to the precise information that provides about the sample, this technique has taken a big importance for chemical analysis and characterization. ${ }^{[69]}$ The main drawback of this technique lays in the weak intensity of the Raman signal. To overcome this disadvantage, some methods such as surface enhanced Raman scattering (SERS) have been proposed. SERS amplifies the Raman signal by several orders of magnitude, ${ }^{[69,70]}$ enabling its use in very different applications.

The successful combination of Raman spectroscopy with EC techniques enables its use in dynamic systems with a high time resolution. Raman-SEC is a powerful technique because it combines the best of two worlds. On the one hand, EC allows the conversion of an electroactive species or the modification of a surface, which could promote a change in the Raman signal. On the other hand, Raman spectra give a clear and unambiguously information about the identity of the materials present on the electrode and its evolution with potential/time, which is particularly important in materials characterization. Usefulness of Raman-SEC is improved by using SERS substrates, being able to detect samples or to follow processes with low amounts of the chemical system studied. Thus, SERS expands the use of Raman-SEC.

Raman-SEC instruments consist of a potentiostat, a laser source, a SEC cell and a spectrometer, Figure 3. The SEC cell must fulfil the conditions to host the typical three-electrode system and the space and suitable environment to carry out the Raman measurement. In the case of Raman spectroscopy, micro-Raman spectrometers, usually confocal microRaman spectrometers, are generally used. Using confocal microscopy, all the information out-of-focus, is removed since the dispersion registered only comes from the plane of focus of the laser. Typical lasers are those belonging to the VIS and the NIR regions. Thus, 532, 633, 785 and $1064 \mathrm{~nm}$ are the most common laser lines. While $532 \mathrm{~nm}$ offers larger cross sections and higher $\mathrm{S} / \mathrm{N}$ ratio, the $785 \mathrm{~nm}$ offers a smaller background signal, which is interesting for measurements with systems with a high fluorescence contribution. Depending on the laser source and the specific problem to be addressed, the spectrometer has to be carefully chosen.

\subsubsection{Raman-SEC Optical Configurations and Cells}

Several configurations of Raman-SEC setups can be found in literature. ${ }^{[39,41]}$ A widely used configuration for RamanSEC is the combination of a micro-Raman spectrometer, usually a confocal microscope coupled to a spectrometer, and a potentiostat. In this setup, three optical configurations (Figure 4.i, 4.j and 4.k) can be used and a very small region of the electrode surface is sampled, obtaining a high spatial resolution. In the normal reflection configuration, Figure 4.i, the WE is inserted in an EC cell, and a thin-layer is created between the optical window and the WE surface to collect as much scattered photons as possible. A thick layer of solution improves the EC response but may worsen the optical signal. Therefore, a compromise between the two responses is required. Using an inverted microscope, Figure 4.k, the laser can sample the electrode/solution interface from below the sample. In this case, there is no problem with the solution adjacent to the electrode, but the use of OTEs is mandatory. In some cases, an immersion objective can be used to obtain the optical response. ${ }^{[71]}$ The angle configuration, Figure 4.j, can be used when Raman measurements are combined with other techniques, as in tip-enhanced Raman spectroscopy ${ }^{[72]}$ (TERS).

It must be noted that a confocal micro-Raman is not always needed. In fact, the use of low-resolution Raman spectrometers has demonstrated to be suitable in the study of different interfacial systems. ${ }^{[73-75]}$ Thus, the use of low-resolution Raman spectrometers coupled with Raman probes with a relatively low magnification allows us to sample non-perfect flat surfaces. Moreover, measurements can be performed without a flat optical window, ensuring a good focus of the laser beam and obtaining good results, with the experiment being performed just in a few-microliters solution drop. ${ }^{[73-75]}$ New Raman-SEC instruments, Figure 7, are similar in size with the UV/VIS-SEC equipment shown in Figure 6. Additionally, Raman-SEC cells can be simplified by using Raman probes, with the type of experimental setup being comparable to the setups used for PL-SEC.

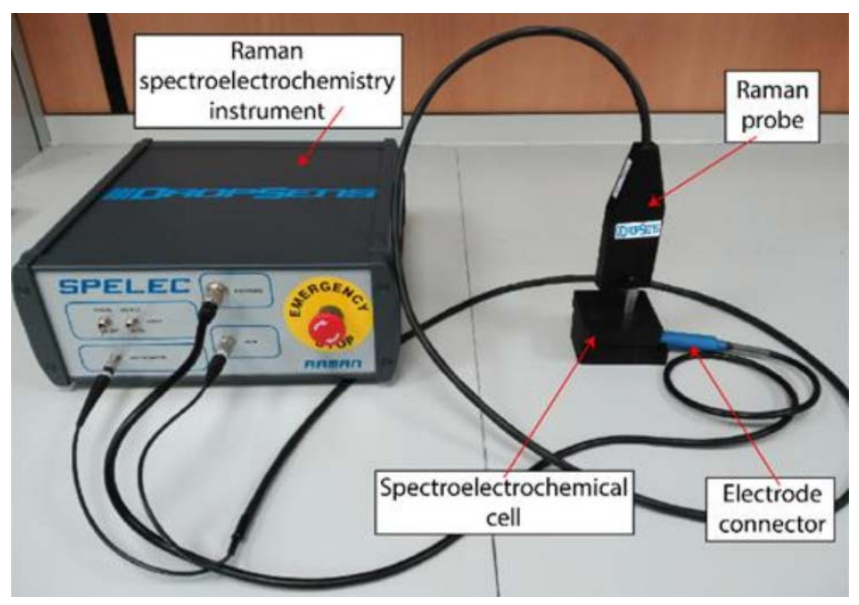

Figure 7. Picture of the instrumentation employed for Raman spectroelectrochemistry with screen-printed electrodes. Reprinted from Ref. ${ }^{[74]}$. Copyright 2018, with permission from Elsevier.

\section{Advantages in Use}

The reversible hypsochromic shift in the absorption onset of a CdS film immobilized on an OTE at cathodic potentials was observed in 1989 by Liu and Bard, ${ }^{[76]}$ performing for the first time a SEC experiment to obtain information about this semiconductor film.

Electrochemistry can be used to determine the number of charges injected into QDs, but there is no distinction between charges injected into delocalized quantum confined states or placed into localized trap states. Simultaneous monitoring of the evolution of the spectra in both the VIS and the IR regions can unambiguously confirm the injection of electrons into quantum confined states of colloidal semiconductor 
NCs. ${ }^{[77-80]}$ For example, the injection of electrons into the quantum-confined states of the CdSe QDs produces an electrochromic response, including a strong, sizetunable, midinfrared (mid-IR) absorption corresponding to an intraband transition, a bleach of the visible interband exciton transitions, and a quench of the narrow band-edge PL. ${ }^{[77,79]}$ Electrons and holes can be injected from an electrode into the quantum confined states of a semiconductor NC film, with the hole injection being less robust than electron injection. ${ }^{[78]}$ SEC can also be used to obtain energy level diagrams. ${ }^{[81]}$ A number of SEC studies of QDs are focused on charge injection into quantum confined band levels of easily reduced QDs with relatively positive conduction-band (CB) potentials (e.g., CdSe, $\mathrm{CdS}, \mathrm{ZnO}, \mathrm{PbSe}){ }^{[65,77,78,80,82-98]}$ Electron injection into the CBs of colloidal chalcogenide QDs bleaches the first excitonic absorption $\left(1 \mathrm{~S}_{\mathrm{h}} \rightarrow 1 \mathrm{~S}_{\mathrm{e}}\right)$, introduces new absorption due to intra-conduction-band $\left(1 \mathrm{~S}_{\mathrm{e}} \rightarrow 1 \mathrm{P}_{\mathrm{e}}\right)$ electronic excitation and diminishes excitonic PL quantum yield through electron-exciton Auger recombination. ${ }^{[65,77-80,82-86,93,95,97,98]}$ SEC can determine the types of surface states in QDs and imitate the evolution of surface defects, providing more dimensional tests for the study of photo-stability. ${ }^{[99,100]}$ The absolute energetic position of the trap levels involved in fluorescence can be determined using PLSEC. ${ }^{[101]}$ The effect of counterions in the PL of CdSe/ZnS QD films was also studied by PL-SEC. ${ }^{[102]}$ In this work, addition of $\mathrm{KCl}$ provokes the shifting of the PL bands of the negatively charged films.

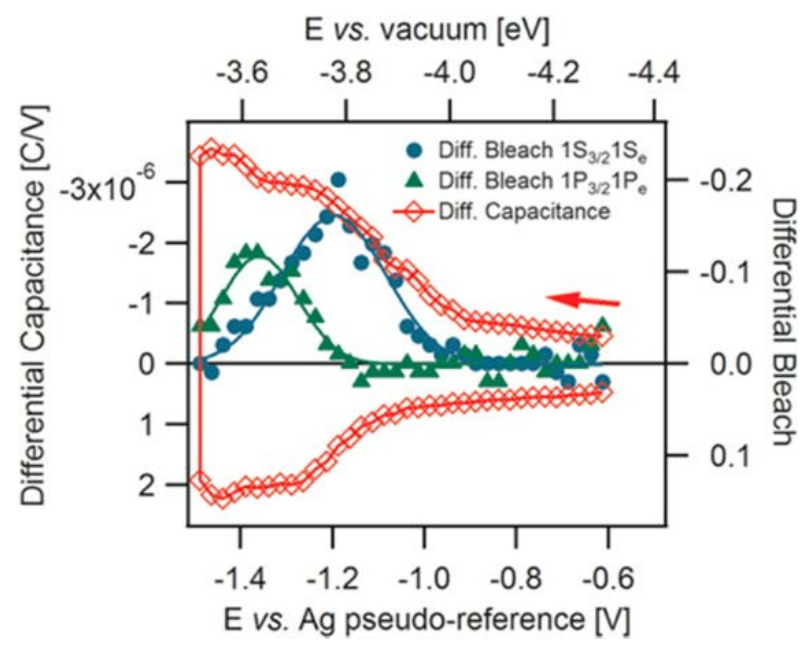

Figure 8. Determination of electron energy levels in a film of 8 $\mathrm{nm}$ CdSe QDs with 8DA ligands by means of differential capacitance $\Delta Q(V) / \Delta V$ (open diamonds) and differential absorption bleach $\Delta\left[\Delta \mathrm{A}_{\mathrm{i}}(\mathrm{V})\right] / \Delta \mathrm{V}$ at transitions involving the $1 \mathrm{~S}_{\mathrm{e}}$ level (closed circles) and $1 \mathrm{P}_{\mathrm{e}}$ level (closed triangles), respectively. Gaussian fits to the differential bleaches are displayed as continuous lines. The electrolyte is $0.1 \mathrm{M} \mathrm{LiClO}_{4}$ in acetonitrile. Reprinted with permission from Ref. ${ }^{[103]}$ Copyright 2018 American Chemical Society.
The knowledge of the absolute energy levels of QDs films is very important for the design of devices based on these nanomaterials. As has been stated above, electrochemical charge injection has been proposed to be very useful to determine absolute energy levels of QD films ${ }^{[103,104]}$ and, using SEC, the simultaneous change in optical absorption of the film allows the quantification of the number of charges in quantumconfined levels and thereby their energetic position. As an example, Houtepen et al. reported electrochemical charge injection of up to 5.5 electrons per QD, yielding the absolute energetic position of both $1 \mathrm{~S}_{\mathrm{e}}$ and $1 \mathrm{P}_{\mathrm{e}}$ electron levels in CdSe QD films, ${ }^{[103]}$ Figure 8. In this work, authors affirm that SEC serves as a tool to sense and control electrostatic interactions with the electrolyte, illuminating the route towards an optimized design of future optoelectronic devices such as QD sensitized solar cells.

Guyot-Sionnest et al. demonstrated the electrochemical control of the PL efficiency of single CdSe/CdS and CdSe/ZnS QDs. ${ }^{[87]}$ A strong PL quenching due to the efficient energy transfer between the QDs and a bare ITO electrode was observed. They solved this problem by adding a spacer layer of $\mathrm{ZnO}$ NCs, obtaining bright $\mathrm{PL}$ with typical blinking behaviour in the neutral state and the PL is reversibly quenched at cathodic potentials due to electron injection. ${ }^{[87]}$ This methodology was used to carry out a controllable charging at the single dot level by applying an electrochemical potential to single $\mathrm{CdSeS} / \mathrm{ZnS}$ and CdSe/ZnS QDs, and the PL lifetimes were monitored by time correlated photon counting with a pulsed excitation. ${ }^{[90]}$ This work showed that ternary dot structures allow the achievement of longer trion lifetimes compared to binary dots of similar sizes. GuySionnest et al. also used PL and absorption mid-IR and NIR-SEC to determine the sign of the carrier, verify the quantum confinement of HgS NCs, determine the bandgap, and finally, understand the mechanism of doping. ${ }^{[105]}$

IR-SEC has also been applied to study the degree of splitting and relative intensity of the intraband features of HgTe QDs, which remain constant across doping levels up to two electrons per QD, ${ }^{[106]}$ Figure 9. This work showed a robust splitting of the intraband absorbance into three peaks across many monodisperse QD samples with different confinement and doping levels. SEC reveals that the peak spacing and relative oscillator strengths of the three peaks do not change appreciably with doping level.

Jeong et al. ${ }^{[107]}$ presented steady-state intraband transitions of $\mathrm{Ag}_{2} \mathrm{Se} \mathrm{NCs}$, using IR-SEC measurements to identify the origin of the electronic transition by finding the correlation between the band-gap transition and the intraband transition. 
a

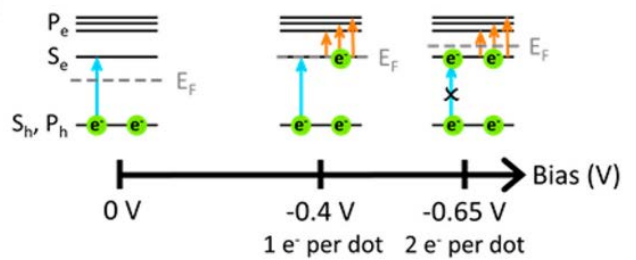

b
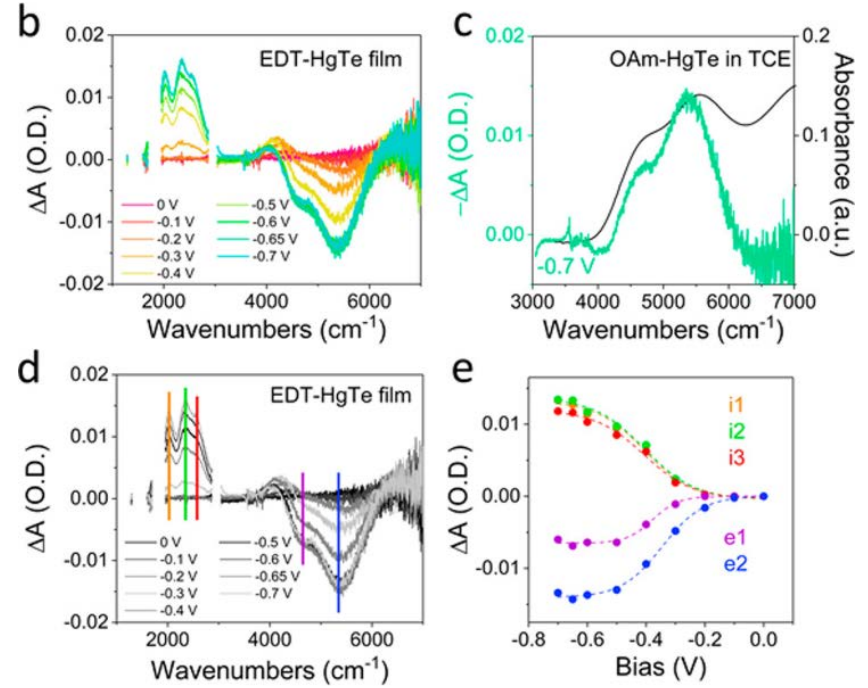

e

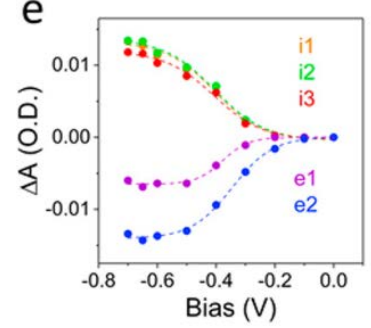

Figure 9. (a) Illustration of the change in the Fermi level (EF) with applied negative bias and its effect on interband absorbance (blue arrow) and intraband absorbance (orange arrows) of a QD film. (b) Difference spectra for a film of EDTcross-linked $\mathrm{HgTe}$ QDs under a series of biases vs $\mathrm{Ag} / \mathrm{AgCl}$. As a negative bias is applied, an intraband absorbance appears, and the first two excitonic features are bleached. (c) The bleach in spectroelectrochemistry matches the energy of the first two excitonic features in a solution absorbance measurement of the as-synthesized HgTe QDs. (e) The change in absorbance for three intraband transitions (i1-i3) and two excitonic bleaches (e1, e2) as a function of applied bias vs $\mathrm{Ag} / \mathrm{AgCl}$ and their corresponding sigmoidal fits, with the position of each transition marked in (d) with the corresponding color. Reprinted with permission from Ref. ${ }^{[06]}$ Copyright 2018 American Chemical Society.

Potential-modulated absorption spectroscopy (EMAS) was initially used to study the bleaching of submonolayers of CdS NPs on ITO. ${ }^{[108]}$ EMAS has also been employed to determine absolute band edge positions, using a well-defined reference electrode under ambient conditions. ${ }^{[104]}$ In EMAS, a periodic wave potential with certain amplitude is added to a constant potential within the band gap of a semiconductor NP. If the maximum of the potential does not reach the lower conduction band edge, then no change in absorbance is expected. When the amplitude is increased, the fundamental absorption of the semiconductor is quenched due to the occupation of the lowest conduction band states. The simultaneous change in absorbance can be plotted against the corresponding potential applied. Eychmüller et al. developed a bleaching model that yields reproducible band edge energies for spherical CdSe NPs. ${ }^{[104]}$ One of the main advantages of EMAS is that extremely weak signal amplitudes can be detected using lock-in amplification, being possible to investigate submonolayers of particles attached to OTEs. Saavedra et al. determined the rate of electron injection with respect to the dependence of the linker employed to attach the NPs. ${ }^{[86]}$ In this work, an ATR setup was combined with the modulation of the applied potential to study the charge injection processes of a CdSe submonolayer. ${ }^{[86]}$

An interesting SEC approach is the coupling of a spectroscopic technique with in-situ potentiometry. ${ }^{[109,110]}$ Band-edge potentials can be insitu quantified using this approach, without special apparatus or modification of NC surface chemistry. Gamelin et al. used potentiometric SEC to analyse the redox potentials of free-standing colloidal n-type CdSe NCs. ${ }^{[11]}$ The CdSe QDs band-edge potentials were tuned by hundreds of millivolts by modifying the QD surface composition, Figure 10. This work shows the importance of NC surface stoichiometry in applications involving tuned NC redox potentials, band-edge alignment, or electron-transfer driving forces. Furthermore, Fermi levels and NC redox potentials were measured using this method. ${ }^{[112]}$ The average number of conduction-band electrons $\left(\mathrm{e}_{\mathrm{CB}}{ }^{-}\right)$per $\mathrm{NC}$ was also assessed using the absorbance. Authors reported extremely slow trapping of $\mathrm{e}_{\mathrm{CB}}{ }^{-}$in free-standing photodoped colloidal n-type CdSe NCs. Potentiometric SEC has also been used to reveal and quantify a fundamental difference between the stabilities of excess valence band holes in colloidal copper-sulfide NC oxidized using $\mathrm{I}_{2}$ or $\mathrm{Fc}^{+} \cdot{ }^{[110]}$

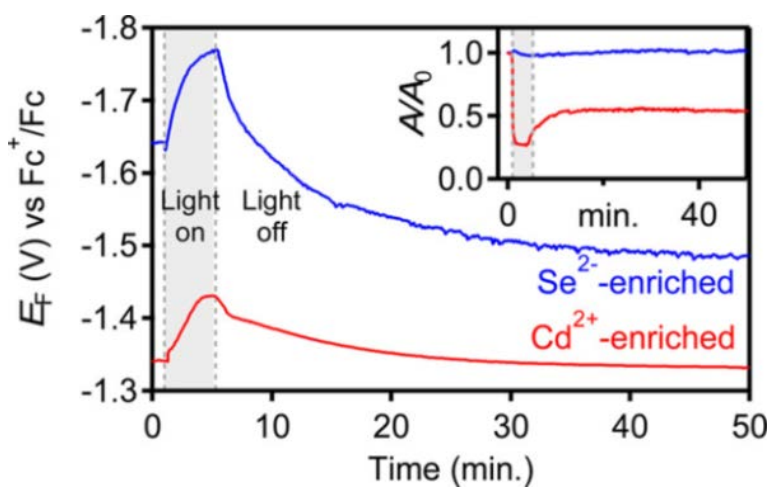

Figure 10. Transient potentiometric data for photodoping $\mathrm{d}=$ $3.8 \mathrm{~nm} \mathrm{Cd}{ }^{2+}$-enriched (red) and $\mathrm{Se}^{2-}$-enriched (blue) CdSe NCs with $\mathrm{Na}\left[\mathrm{Et}_{3} \mathrm{BH}\right]$ as the hole quencher in a THF solution with 0.1 $M$ TBAPF $_{6}$ as the supporting electrolyte. For each sample, the NCs were photodoped using a $405 \mathrm{~nm}$ diode $(50 \mathrm{~mW})$ between $\mathrm{t}=1$ and $5 \mathrm{~min}$. At $\mathrm{t}=5 \mathrm{~min}$, photoexcitation was stopped, but $E_{F}$ was monitored further. Inset: A/AO collected in concert with $E_{F}$. Reprinted with permission from Ref. ${ }^{[112]}$ Copyright 2018 American Chemical Society.

Combination of SEC, differential capacitance and source-drain electronic conductance measurements was used to determine both the mobility of electrons out-ofplane (moving perpendicular through a $\mathrm{ZnO}$ QD film) during charge injection and the mobility of electrons inplane (moving parallel to the substrate) in a sourcedrain configuration. The out-of-plane electron mobility was 7 orders of magnitude lower than the in-plane one. ${ }^{[113]}$

PL-SEC has been used to probe the distribution of Cu-related trap states responsible for PL of $\mathrm{CuInS}_{2}$ NCs. ${ }^{[59]} \mathrm{PL}$ line width is correlated to the width of the "bright" $\mathrm{Cu}^{+}$trap-state distribution in the ensemble. PL can be tuned by electron injection or removal. PL peak 
position is red-shifted in the case of electron injection and blue-shifted when electrons are withdrawn. Moreover, PL intensity increases when electrons are injected, whereas it decreases and becomes narrower when electrons are withdrawn. Efficient hole trapping can be activated by injecting electrons into the "dark" defect states, $\mathrm{Cu}^{2+}$. These observations contrast to the observations of Klimov et al. ${ }^{[114]}$ using also PL-SEC, who reported the presence of both active $\mathrm{Cu}^{+} \mathrm{Cu}^{2+}$ and luminescent centers in the ground state, depending on the Cu-to-In ratio in the $\mathrm{CuInS}_{2} \mathrm{NCs}$.
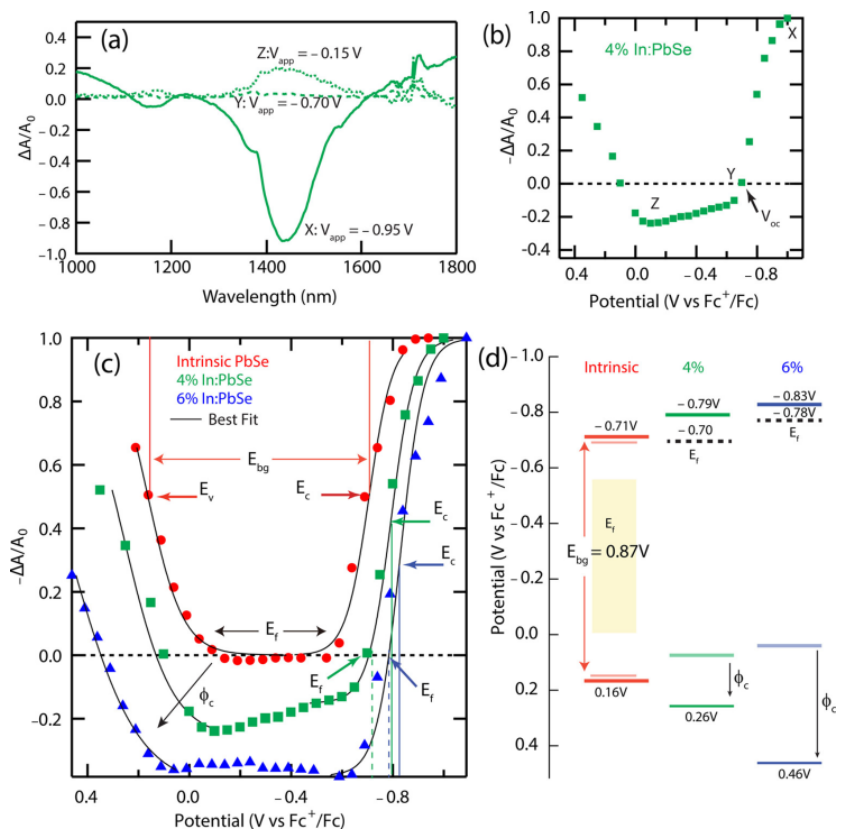

Figure 11. (a) Normalized differential absorption spectra of $d=$ $4.3 \mathrm{~nm} \mathrm{4 \%} \mathrm{In:PbSe} \mathrm{QDs} \mathrm{deposited} \mathrm{as} \mathrm{a} \mathrm{thin} \mathrm{film} \mathrm{on} \mathrm{FTO} \mathrm{in} 0.1$ $\mathrm{M} \mathrm{LiClO}_{4}$ in acetonitrile. The spectra were collected under electrochemical bias at $\mathrm{V}_{\mathrm{App}}=-0.95 \mathrm{~V}$ (solid), $-0.70 \mathrm{~V}$ (dashed), and $-0.15 \mathrm{~V}$ (dotted) in a three-electrode configuration. (b) Differential excitonic absorption at $1450 \mathrm{~nm}$ of $4 \% \mathrm{In}: \mathrm{PbSe} \mathrm{QD}$ thin films in $0.1 \mathrm{M} \mathrm{LiClO}_{4}$ in acetonitrile as a function of the electrochemical bias. $X, Y, Z$ points correspond to $\mathrm{V}_{\text {App }}=-0.95 \mathrm{~V},-0.70 \mathrm{~V}$, and $-0.15 \mathrm{~V}$, respectively. (d) Normalized differential excitonic absorption of intrinsic $\mathrm{PbSe}$ (red-circles), 4\% In:PbSe (green-squares), and 6\% In:PbSe (blue-triangles) QD thin films as a function of the electrochemical bias. All potentials are referenced against the experimentally determined Ferrocenium/Ferrocene ( $\mathrm{Fc}^{+} / \mathrm{Fc}$ ) redox couple. (d) Energy diagram of $\mathrm{PbSe} Q \mathrm{QDs}$ with respect to $\mathrm{FC}^{+} / \mathrm{Fc}$ redox couple. The Fermi level shifts to more negative values $(-0.7$ and $-0.78 \mathrm{~V})$ for the 4 and $6 \%$ samples. The difference between $E_{f}$ and the conduction band is smaller for the $6 \%$ sample; $\left(\Delta E_{f}=E_{c}-E_{f}\right)$, and $\Delta E_{f}$ is $0.09 V$ for the $4 \%$ sample, and $0.05 \mathrm{~V}$ for the $6 \%$ sample. The $E_{f}$ of intrinsic $\mathrm{PbSe}$ QDs can only be specified in our experiment with an upper limit of $\sim-0.55 \mathrm{~V}$ and lower limit of $\sim 0.0 \mathrm{~V}$ (shaded area for intrinsic PbSe QDs). The onset for hole-injection into the valence band, increases by $\sim 300 \mathrm{mV}$ from intrinsic $\mathrm{PbSe}$ to $6 \% \mathrm{In}: \mathrm{PbSe}$ (denoted as $\phi c$ ). Overall, increasing In content in PbSe QDs leads to more n-type character (smaller $\Delta \mathrm{E}_{\mathrm{f}}$ ). Adapted with permission from Ref. ${ }^{[115]}$ Copyright 2018 American Chemical Society.

Houtepen et al. used UV/VIS-SEC and PL-SEC to study CdTe NC films, observing a strong increase in the PL intensity as the Fermi level is raised electrochemically and trap states in the bandgap become occupied with electrons. ${ }^{[58]}$ Houtepen et al. have also shown that electron trapping in QD films can be slowed down by 3 orders of magnitude by electrochemically filling trap states. ${ }^{[116]}$ Later, they extended these studies with ultrafast transient absorption and time-resolved PLSEC experiments to determine the electron and hole trapping rates of CdTe QDs as a function of the Fermi level. ${ }^{[117]}$

The influence of trap electrochemistry on wide-gap semiconductor NC photoluminescence can be examined using UV/VIS-SEC and PL-SEC. Large PL was observed in $\mathrm{ZnSe}$ and $\mathrm{Mn}^{2+}$-doped ZnSe NCs upon application of a reducing potential and is reversed upon return to the equilibrium potential. The electrobrightening was related to the transfer of electrons into NC films, implicating reductive passivation of midgap surface electron traps. ${ }^{\text {[93] }}$

UV/VIS-SEC was used to demonstrate characteristic n-type signatures of $\mathrm{In}^{3+}$ doped PbSe QDs, Figure 11, including both an induced absorption within the electrochemical bandgap and a shift of the Fermi level toward the conduction band. ${ }^{[115]}$

PL-SEC experiments on Cu-doped core/shell $\mathrm{ZnSe} / \mathrm{CdSe}$ NCs were used to probe the PL intensity and branching ratio between the band edge and the $\mathrm{Cu}$ related emission channels as a function of controlled activation and deactivation (passivation) of surface traps. By raising/lowering the Fermi level, the occupancy of surface trap sites can be modified. Thus, the overall emission intensity as well as the ratio between the intensities of the band edge and $\mathrm{Cu}$-related features can be reversibly tuned. ${ }^{[118]}$ SEC experiments obtained with $\mathrm{Mn}^{2+}$-doped CdS NCs provide convincing evidence against any fundamental role played by surface trap states in the electrochemical PL quenching of these NCs. ${ }^{[88]}$ This conclusion is consistent with previous demonstrations that surface trapped electrons are unable to effectively quench the excitonic luminescence of undoped QDs. ${ }^{[84]}$

PL blinking, random switching between states of high-low emissivities, is a universal property of emitters such as QDs. Klimov et al. used SEC to reveal the existence of two types of blinking mechanism: ${ }^{[89]}$ (i) conventional blinking due to charging and discharging of the NC core, in which lower PL intensities correlate with shorter PL lifetimes, Figure 12; and (ii) in which large changes in the emission intensity are not accompanied by significant changes in emission dynamics.

UV/VIS-SEC was used to determine the CdSe-PbSe QD energy level offset in a heterojunction comprised of both materials. CdSe and PbSe QDs feature different band gaps, therefore electrons occupying the eigenstates of CdSe from those in PbSe QDs can be resolved. ${ }^{[119]}$

Colloidal “dot-in-bulk” NCs (DiB NCs) consist of a quantum confined core embedded into a bulk-like shell of a larger energy gap that are capable of producing tunable two-colour emission. PL-SEC has been used to elucidate the effect of various trapping mechanisms on core- and shell-emission channels in CdSe/CdS DiB NCs. ${ }^{[120]}$ In this work, it is shown that the core emission was only weakly affected by the defects at the surface of the CdS shell, whereas the shell emission was highly sensitive to the changes in the activity of surface traps. 
SEC experiments in a controlled atmosphere have demonstrated the ability of colloidal quantum wells to probe oxidative species through brightening of their PL. ${ }^{[121]}$
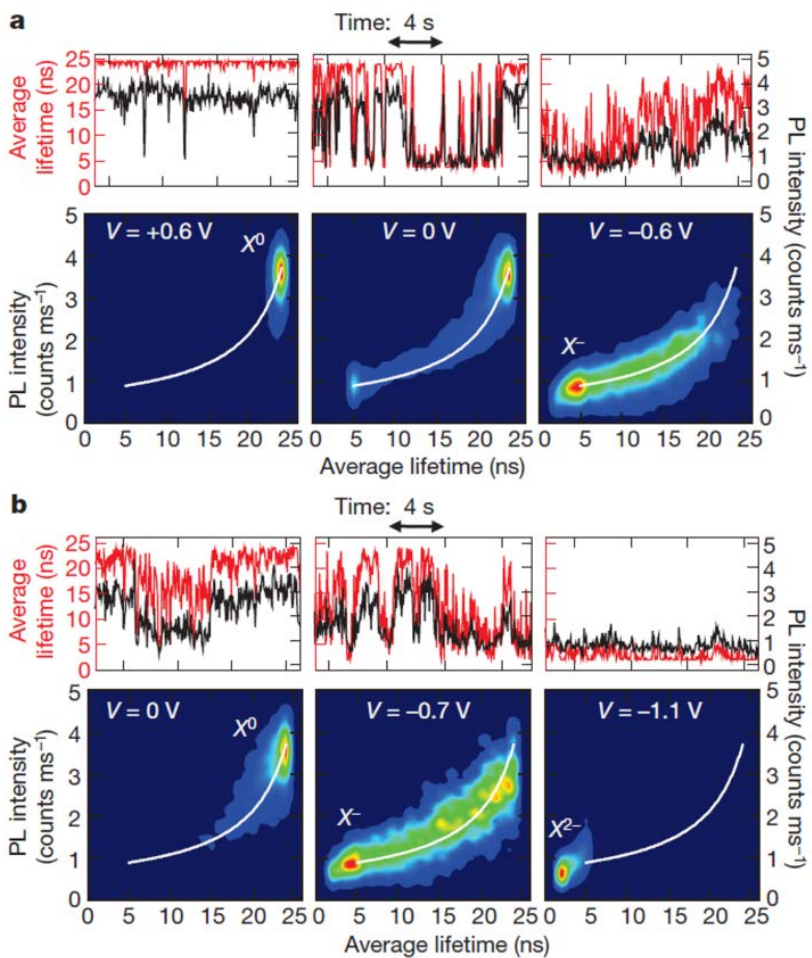

Figure 12. Correlated photoluminescence intensity and lifetime fluctuations: A-type blinking and flickering. a Photoluminescence intensities (black lines) and average lifetimes (red lines), and corresponding fluorescence lifetimeintensity distributions (FLIDs), for the nanocrystal at three different potentials. Binary blinking seen at $V=0 \mathrm{~V}$ is largely suppressed at $V=+0.6 \mathrm{~V}$, whereas electron injection is achieved at $V=-0.6 \mathrm{~V}$. In the FLID colour scale, red corresponds to the most frequently occurring lifetime-intensity pair, and probabilities less than $1 \%$ of this maximum are indicated by dark blue. A linear scaling from blue to red is used between these extremes. b, Data from the same nanocrystal, acquired on a different day, display continuous photoluminescence intensity and lifetime fluctuations, typical of flickering. At $V=-1.1 \mathrm{~V}$, we observe emission from a doubly charged exciton, $X^{2-}$. All data were analysed with a bin size of $50 \mathrm{~ms}$.. Reprinted by permission from Springer Nature: Nature from Ref. ${ }^{\left[{ }^{[9]}\right.}$ Copyright 2011.

Fluorescence enhancement/quenching in QDs can be electrochemically modulated by controlling electron transfer between attached molecules and QDs. PL-SEC is very useful to demonstrate this point. ${ }^{[122,123]}$ For example, a PL-SEC study demonstrated that the reduced state of coenzyme Q (CoQ) disulfide derivatives enhanced the fluorescence intensity of CdTe/ZnS QDs, while the oxidized state of the CoQ conjugates quenched the fluorescence to different degrees. ${ }^{[123]}$ Electrochromic dyes have been used to modulate the luminescence quenching of QDs, tuning at the redox potential of the dye in the hybrid film. ${ }^{\text {122] }}$ FRET has been widely investigated by SEC. ${ }^{[124-126]}$ In the case of QDs, for example, electrochromic fluorescence switching of Prussian Blue/CdTe films were investigated using SEC ${ }^{[127]}$. The absorption spectrum of $\mathrm{PB}$ and the emission spectrum of CdTe QDs are ideally overlapped allowing the electrochemical control of the fluorescence intensity of the QDs by FRET, Figure 13. SEC modulation of the dichlorophenolindophenol sodium salt QDs (DCIP-QDs) and cysteamine capped CdTe QDs (CA-CdTe QDs) was performed in order to change the redox state of the dye and further modulate the fluorescence intensity of the CA-CdTe QDs. DCIPCA-CdTe QDs were used for the detection of ascorbic acid. ${ }^{[128]}$

Elucidation of the electronic structure of organic/inorganic NCs can be complex. SEC can help to assign electron-transfer processes. For example, in a surface-functionalized $\mathrm{PbS} \mathrm{NCs}$ with 5,5"-dithiol$\left[2,2^{\prime}: 5,2^{\prime \prime}\right.$-terthiophene] (T3DSH) a reduction peak was observed in cyclic voltammetry (CV) that should not be related to the molecular frontier of T3DSH. UV/VISSEC was used to elucidate the provenance of this unassigned reduction wave. ${ }^{[129]}$

Combination of confocal fluorescence microscopy, EC, and SEIRAS, was used to study the mechanisms of membrane penetration by $\mathrm{CdSe} / \mathrm{ZnS}$ core/shell QDs coated with the zwitterionic thiol ligand Dpenicillamine (DPA-QDs). Spectroscopy revealed the interactions of DPA-QDs with model lipid bilayers, while electrochemistry demonstrated that penetration of DPA-QDs into the lipid membranes did not lead to the formation of (ion-conducting) pores. ${ }^{[130]}$

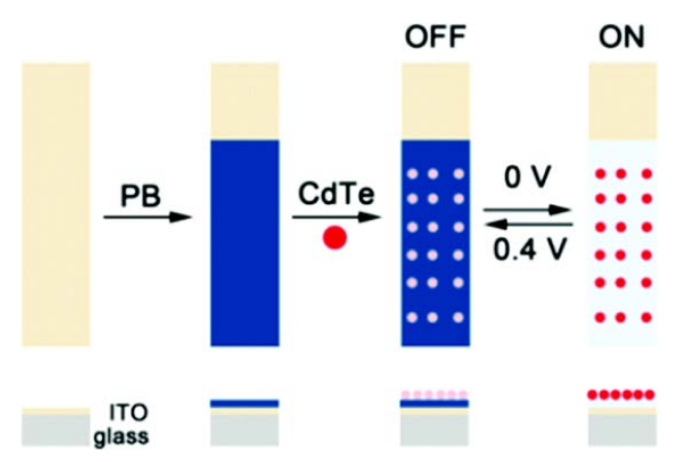

Figure 13. Schematic diagram of the fluorescent switch assembly. Reprinted with permission from Ref. ${ }^{[127]}$. Copyright 2019, The Royal Society of Chemistry.

Raman-SEC has been scarcely used to characterize QDs. In most cases it has been used to characterize hybrid materials, ${ }^{[131,132]}$ obtaining important information about the organic part of the nanomaterial. Nevertheless, Raman-SEC should be very useful to study QDs because most of these NCs exhibit well-defined phonons. ${ }^{[133-138]}$

SEC has been much more used in the characterization of semiconductor QDs than in the characterization of carbon QDs. Nevertheless, some examples can be found in literature. Carbon nanodots (CNDs) were studied using PL-SEC, demonstrating that the excitation dependent fluorescence of the CNDs may be attributed to a combination of the core $\mathrm{C}=\mathrm{C}$, surface functionalities $(\mathrm{C}=\mathrm{O}, \mathrm{C}-\mathrm{O}$, and $\mathrm{COOH})$, and/or surface electronic state transitions. ${ }^{[139]}$

Changes in the optical properties of GQDs during electrochemical reduction and oxidation were investigated by PL-SEC, providing a direct in-situ evidence of the dependence of GQDs luminescence on their redox state. ${ }^{[52]} \mathrm{QY}$ increased/decreased and PL 
emission blue/red-shifted upon GQDs reduction/oxidation, Figure 14, rendering information about electronic transitions involved in the redox processes. UV/VIS-SEC was also used to study the electronic structure of a series of bottom-up synthesized GQDs smaller than $2 \mathrm{~nm} .^{[140]}$ This work showed that for strongly confined GQDs, dependence of the band gap on the size of GQDs deviates from the prediction of the standard Dirac fermion model, but agrees well with the models explicitly accounting for the electron-electron interactions.

UV/VIS-SEC can be used to induce spectral overlapping between redox-dependent quenching molecules and biomass carbon QDs. Using this strategy, a fluorescence quenching consistent with a FRET mechanism has been observed, ${ }^{[141]}$ although an electron transfer mechanism could not be excluded.
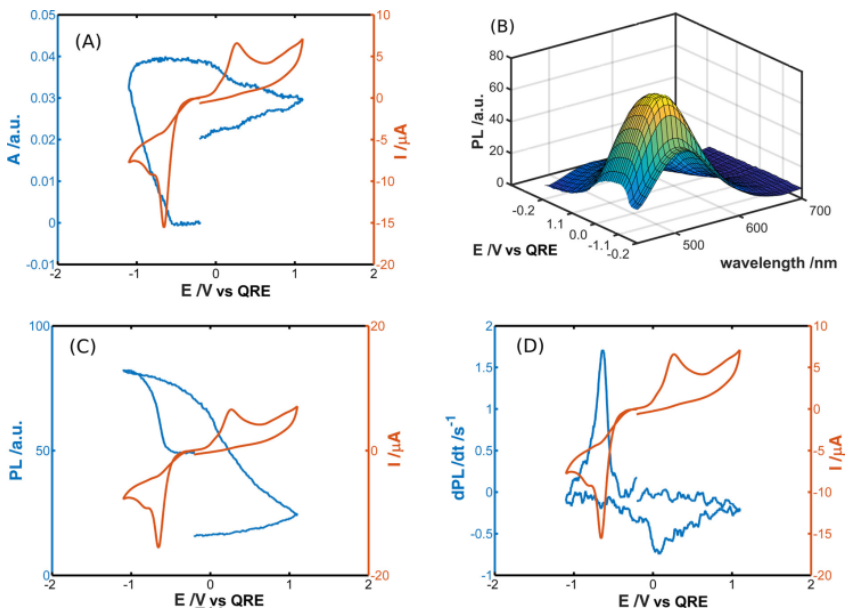

Figure 14. (A) CV (brown line) and VA at $385 \mathrm{~nm}$ (blue line); (B) evolution of the PL emission spectra obtained during the cyclic voltammetry; (C) CV (brown line) and PL at $500 \mathrm{~nm}$ (blue line); (D) CV (brown line) and derivative of PL intensity at 500 $\mathrm{nm}$ (blue line) recorded during the potential scan carried out in a $0.00145 \mathrm{mg} \mathrm{mL}^{-1} \mathrm{GQD}$ solution in $0.1 \mathrm{M} \mathrm{LiClO}_{4}$. $\mathrm{E}_{\mathrm{i}}=\mathrm{E}_{\mathrm{f}}=$ $-0.20 \mathrm{~V}, \mathrm{E}_{\mathrm{v} 1}=-1.10 \mathrm{~V}, \mathrm{E}_{\mathrm{v} 2}=+1.10 \mathrm{~V}, \mathrm{v}=0.010 \mathrm{~V} \mathrm{~s}^{-1}$, integration time $=200 \mathrm{~ms}$. Reprinted with permission from Ref. ${ }^{[52]}$ Copyright 2017 American Chemical Society.

\section{Outlook}

As has been stated in the previous section, the different SEC techniques analysed in this review provide a lot of information about QDs. In our opinion, the limited use of SEC in this field is probably due to the complex experimental setups, together with the high amount of data obtained in a single experiment. Moreover, the very expensive instruments and the complex optical configurations can scare potential users. However, for many applications, SEC is actually a very simple technique and SEC experiments can be performed using simple setups and low-cost instrumentation, as has been demonstrated in a number of works. Sometimes, interpretation of results can be complex due to the high amount of information of different nature that is obtained in a single experiment. However, information is a basis for knowledge. Once demonstrated that SEC is very useful and versatile to study these nanomaterials, a great effort must be made to simplify the experimental setups, allowing non-expert users to perform experiments in an easy way. Nowadays, new instruments are commercially available, some of them including software for the acquisition and treatment of the data. Some SEC cells, apart from the typical platinum mesh that is not useful for QDs, are also commercially available. Nevertheless, development of new simple cells would help to spread the use of this set of techniques for the study of QDs. We hope that in a near future, SEC becomes a routine technique for the characterization not only of QDs but also of many other nanomaterials, because it provides a huge amount of information about their properties.

Finally, it should be highlighted that new technical developments are needed to obtain high-quality SEC responses. Better light sources, optical fibers and detectors should help to improve the $\mathrm{S} / \mathrm{N}$ ratio and to obtain better results in a simple way. Furthermore, the power of SEC is increased when it is combined with other in-situ techniques ${ }^{[142-144]}$ or when different spectral techniques are simultaneously coupled ${ }^{[145]}$ in a single experiment. Multiresponse techniques should be very important in the future for the characterization of materials.

\section{Acknowledgements}

Authors acknowledge the financial support from Ministerio de Economía y Competitividad (Grants CTQ2017-83935-RAEI/FEDERUE) and Junta de Castilla y León (Grant BU033-U16 and BU297P18). J.G-R.. thanks UBU for his postdoctoral contract. J.V.P-R. thanks JCyL for his postdoctoral fellowship (Grant BU033-U16). Thanks to J. López-Palacios, because he started to work in spectroelectrochemistry in a small city like Burgos and he taught us all we know now.

\section{References}

[1] Z. Zhang, J. Zhang, N. Chen, L. Qu, Energy Environ. Sci. 2012, 5, 8869-8890.

[2] E. Roduner, Chem. Soc. Rev. 2006, 35, 583-592.

[3] M. Bacon, S. J. Bradley, T. Nann, Part. Part. Syst. Charact. 2014, 31, 415-428.

[4] A. P. Alivisatos, Science 1996, 271, 933-937.

[5] C. Burda, X. Chen, R. Narayanan, M. A. El-Sayed, Chem. Rev. 2005, 105, 1025-1102.

[6] A. D. Yoffe, Adv. Phys. 2001, 50, 1-208.

[7] D. Bera, L. Qian, T.-K. Tseng, P. H. Holloway, Materials 2010, 3, 2260-2345.

[8] P. Tian, L. Tang, K. S. Teng, S. P. Lau, Mater. Today Chem. 2018, 10, 221-258.

[9] S. Y. Lim, W. Shen, Z. Gao, Chem. Soc. Rev. 2015, 44, 362-381.

[10] Y. Xu, X. Wang, W. L. Zhang, F. Lv, S. Guo, Chem. Soc. Rev. 2018, 47, 586-625.

[11] A. P. Litvin, I. V. Martynenko, F. Purcell-Milton, A. V. Baranov, A. V. Fedorov, Y. K. Gun'Ko, J. Mater. Chem. A 2017, 5, 13252-13275.

[12] I. V. Martynenko, A. P. Litvin, F. Purcell-Milton, A. V. Baranov, A. V. Fedorov, Y. K. Gun'ko, J. Mater. Chem. B 2017, 5, 6701-6727.

[13] A. P. Alivisatos, J. Phys. Chem. 1996, 100, 1322613239.

[14] C. de M. Donegá, Chem. Soc. Rev. 2011, 40, 15121546.

[15] T. Trindade, P. O’Brien, N. L. Pickett, Chem. Mater. 2001, 13, 3843-3858.

[16] L. Jing, S. V. Kershaw, Y. Li, X. Huang, Y. Li, A. L. Rogach, M. Gao, Chem. Rev. 2016, 116, 10623-10730. 
[17] J. Li, J. Z. Zhang, Coord. Chem. Rev. 2009, 253, 3015-3041.

[18] Y. E. Panfil, M. Oded, U. Banin, Angew. Chemie Int. Ed. 2018, 57, 4274-4295.

[19] J. Y. Kim, O. Voznyy, D. Zhitomirsky, E. H. Sargent, Adv. Mater. 2013, 25, 4986-5010.

[20] D. Bera, L. Qian, P. H. Holloway, in Lumin. Mater. Appl., John Wiley \& Sons, Ltd, Chichester, UK, 2008, pp. 19-73.

[21] W. W. Yu, L. Qu, W. Guo, X. Peng, Chem. Mater. 2003, 15, 2854-2860.

[22] A. Kitai, Ed. , Materials for Solid State Lighting and Displays, John Wiley \& Sons, Ltd, Chichester, UK, 2017.

[23] K. Chou, A. Dennis, Sensors 2015, 15, 13288-13325.

[24] A. P. Alivisatos, W. Gu, C. Larabell, Annu. Rev. Biomed. Eng. 2005, 7, 55-76.

[25] A. M. Schimpf, K. E. Knowles, G. M. Carroll, D. R. Gamelin, Acc. Chem. Res. 2015, 48, 1929-1937.

[26] M. Amelia, C. Lincheneau, S. Silvi, A. Credi, Chem. Soc. Rev. 2012, 41, 5728-5743.

[27] A. Franceschetti, A. Zunger, Phys. Rev. B 2000, 62, 2614-2623.

[28] M. Amelia, T. Avellini, S. Monaco, S. Impellizzeri, I. Yildiz, F. M. Raymo, A. Credi, Pure Appl. Chem. 2011, 83, 1-8.

[29] P. Guyot-Sionnest, Microchim. Acta 2008, 160, 309314.

[30] M. V. Kovalenko, L. Manna, A. Cabot, Z. Hens, D. V. Talapin, C. R. Kagan, V. I. Klimov, A. L. Rogach, P. Reiss, D. J. Milliron, P. Guyot-Sionnnest, G. Konstantatos, W. J. Parak, T. Hyeon, B. A. Korgel, C. B. Murray, W. Heiss, ACS Nano 2015, 9, 1012-1057.

[31] Y. Barak, I. Meir, A. Shapiro, Y. Jang, E. Lifshitz, Adv. Mater. 2018, 30, 1801442.

[32] D. Mo, L. Hu, G. Zeng, G. Chen, J. Wan, Z. Yu, Z. Huang, K. He, C. Zhang, M. Cheng, Appl. Microbiol. Biotechnol. 2017, 101, 2713-2733.

[33] N. Zhang, L. Zhang, Y.-F. Ruan, W.-W. Zhao, J.-J. Xu, H.-Y. Chen, Biosens. Bioelectron. 2017, 94, 207-218.

[34] X. Chen, Y. Liu, Q. Ma, J. Mater. Chem. C 2018, 6, 942-959.

[35] Z. Fan, S. Li, F. Yuan, L. Fan, RSC Adv. 2015, 5 19773-19789.

[36] T.-F. Yeh, W.-L. Huang, C.-J. Chung, I.-T. Chiang, L.-C. Chen, H.-Y. Chang, W.-C. Su, C. Cheng, S.-J. Chen, H. Teng, J. Phys. Chem. Lett. 2016, 7, 2087-2092.

[37] P. Zheng, N. Wu, Chem. - An Asian J. 2017, 12, 23432353.

[38] S. Bak, D. Kim, H. Lee, Curr. Appl. Phys. 2016, 16, 1192-1201.

[39] L. León, J. D. Mozo, TrAC Trends Anal. Chem. 2018, 102, 147-169.

[40] T. E. Keyes, R. J. Forster, in Handb. Electrochem., Elsevier, 2007, pp. 591-635.

[41] Y. Zhai, Z. Zhu, S. Zhou, C. Zhu, S. Dong, Nanoscale 2018, 10, 3089-3111.

[42] N. González-Diéguez, A. Colina, J. López-Palacios, A. Heras, Anal. Chem. 2012, 84, 9146-9153.

[43] J. Garoz-Ruiz, A. Heras, S. Palmero, A. Colina, Anal. Chem. 2015, 87, 6233-6239.

[44] J. López-Palacios, A. Colina, A. Heras, V. Ruiz, L. Fuente, Anal. Chem. 2001, 73, 2883-2889.

[45] T. Campos Hernández, A. C. Fernández Blanco, A. T. Williams, M. Velický, H. V Patten, A. Colina, R. A. W. Dryfe, Electroanalysis 2015, 27, 1026-1034.

[46] J. P. Skully, R. L. McCreery, Anal. Chem. 1980, 52, 1885-1889.

[47] J. Garoz-Ruiz, A. Heras, A. Colina, Anal. Chem. 2017, 89, 1815-1822.

[48] O. Alévêque, E. Levillain, L. Sanguinet, Electrochem. Commun. 2015, 51, 108-112.

[49] P. Audebert, F. Miomandre, Chem. Sci. 2013, 4, 575584.

[50] D. Martín-Yerga, A. Pérez-Junquera, D. HernándezSantos, P. Fanjul-Bolado, Anal. Chem. 2017, 89, 1064910654.
[51] D. Martín-Yerga, A. Pérez-Junquera, M. B. GonzálezGarcía, D. Hernández-Santos, P. Fanjul-Bolado, Anal. Chem. 2018, 90, 7442-7449.

[52] J. Barrera, D. Ibañez, A. Heras, V. Ruiz, A. Colina, J. Phys. Chem. Lett. 2017, 8, 531-537.

[53] S. Bkhach, Y. Le Duc, O. Alévêque, C. Gautier, P. Hudhomme, E. Levillain, ChemElectroChem 2016, 3, 887-891.

[54] A. Yildiz, P. T. Kissinger, C. N. Reilley, Anal. Chem. 1968, 40, 1018-1024.

[55] Y. F. Lee, J. R. Kirchhoff, Anal. Chem. 1993, 65, 3430-3434.

[56] J.-S. Yu, C. Yang, H.-Q. Fang, Anal. Chim. Acta 2000, 420, 45-55.

[57] M. Dias, P. Hudhomme, E. Levillain, L. Perrin, Y. Sahin, F.-X. Sauvage, C. Wartelle, Electrochem. Commun. 2004, 6, 325-330.

[58] W. van der Stam, I. du Fossé, G. Grimaldi, J. O. V. Monchen, N. Kirkwood, A. J. Houtepen, Chem. Mater. 2018, 30, 8052-8061.

[59] W. van der Stam, M. de Graaf, S. Gudjonsdottir, J. J. Geuchies, J. J. Dijkema, N. Kirkwood, W. H. Evers, A. Longo, A. J. Houtepen, ACS Nano 2018, 12, 11244-11253.

[60] F. Miomandre, C. Allain, G. Clavier, J.-F. Audibert, R. B. Pansu, P. Audebert, F. Hartl, Electrochem. Commun. 2011, 13, 574-577.

[61] C. Tourbillon, F. Miomandre, J. F. Audibert, M. Lepeltier, P. Martin, J. C. Lacroix, Electrochim. Acta 2015 $179,618-625$.

[62] J. Shepherd, Y. Yang, D. Bizzotto, J. Electroanal. Chem. 2002, 524-525, 54-61.

[63] L. M. Moretto, T. Kohls, A. Chovin, N. Sojic, P. Ugo, Langmuir 2008, 24, 6367-6374.

[64] C. Lei, D. Hu, E. J. Ackerman, Chem. Commun. 2008, 7345, 5490-5492.

[65] A. K. Gooding, D. E. Gómez, P. Mulvaney, ACS Nano 2008, 2, 669-676.

[66] A. N. Asanov, W. W. Wilson, P. B. Oldham, Anal. Chem. 1998, 70, 1156-1163.

[67] F. Miomandre, E. Lépicier, S. Munteanu, O. Galangau, J. F. Audibert, R. Méallet-Renault, P. Audebert, R. B. Pansu, ACS Appl. Mater. Interfaces 2011, 3, 690-696.

[68] R. L. McCreery, Raman Spectroscopy for Chemical Analysis, John Wiley \& Sons, Inc., Hoboken, NJ, USA, 2000.

[69] B. Sharma, R. R. Frontiera, A. Henry, E. Ringe, R. P. Van Duyne, Mater. Today 2012, 15, 16-25.

[70] S. Schlücker, Angew. Chemie Int. Ed. 2014, 53, 47564795.

[71] Z.-C. Zeng, S. Hu, S.-C. Huang, Y.-J. Zhang, W.-X. Zhao, J.-F. Li, C. Jiang, B. Ren, Anal. Chem. 2016, 88, 9381-9385.

[72] Z.-C. Zeng, S.-C. Huang, D.-Y. Wu, L.-Y. Meng, M.H. Li, T.-X. Huang, J.-H. Zhong, X. Wang, Z.-L. Yang, B. Ren, J. Am. Chem. Soc. 2015, 137, 11928-11931.

[73] J. V. Perales-Rondon, S. Hernandez, A. Heras, A. Colina, Appl. Surf. Sci. 2019, 473, 366-372.

[74] D. Martín-Yerga, A. Pérez-Junquera, M. B. GonzálezGarcía, J. V. Perales-Rondon, A. Heras, A. Colina, D. Hernández-Santos, P. Fanjul-Bolado, Electrochim. Acta 2018, 264, 183-190.

[75] A. Colina, V. Ruiz, A. Heras, E. Ochoteco, E. Kauppinen, J. López-Palacios, Electrochim. Acta 2011, 56, 1294-1299.

[76] C. Y. Liu, A. J. Bard, J. Phys. Chem. 1989, 93, 77497750 .

[77] C. Wang, M. Shim, P. Guyot-Sionnest, Science 2001, 291, 2390-2392.

[78] B. L. Wehrenberg, P. Guyot-Sionnest, J. Am. Chem. Soc. 2003, 125, 7806-7807.

[79] C. Wang, M. Shim, P. Guyot-Sionnest, Appl. Phys. Lett. 2002, 80, 4-6.

[80] M. Shim, P. Guyot-Sionnest, Nature 2000, 407, 981983.

[81] H. Liu, S. Keuleyan, P. Guyot-Sionnest, J. Phys. Chem. C 2012, 116, 1344-1349. 
[82] C. Wang, B. L. Wehrenberg, C. Y. Woo, P. GuyotSionnest, J. Phys. Chem. B 2004, 108, 9027-9031.

[83] D. Yu, B. L. Wehrenberg, P. Jha, J. Ma, P. GuyotSionnest, J. Appl. Phys. 2006, 99, 104315.

[84] P. P. Jha, P. Guyot-Sionnest, J. Phys. Chem. C 2007, $111,15440-15445$.

[85] P. P. Jha, P. Guyot-Sionnest, ACS Nano 2009, 3, 1011-1015.

[86] Z. O. Araci, C. R. Shallcross, N. R. Armstrong, S. S. Saavedra, J. Phys. Chem. Lett. 2010, 1, 1900-1905.

[87] P. P. Jha, P. Guyot-Sionnest, J. Phys. Chem. C 2010, 114, 21138-21141

[88] M. A. White, A. L. Weaver, R. Beaulac, D. R. Gamelin, ACS Nano 2011, 5, 4158-4168.

[89] C. Galland, Y. Ghosh, A. Steinbrück, M. Sykora, J. A. Hollingsworth, V. I. Klimov, H. Htoon, Nature 2011, 479, 203-207.

[90] W. Qin, R. A. Shah, P. Guyot-Sionnest, ACS Nano 2012, 6, 912-918.

[91] A. J. Houtepen, D. Vanmaekelbergh, J. Phys. Chem. B 2005, 109, 19634-19642.

[92] M. J. Greaney, S. Das, D. H. Webber, S. E. Bradforth, R. L. Brutchey, ACS Nano 2012, 6, 4222-4230.

[93] A. L. Weaver, D. R. Gamelin, J. Am. Chem. Soc. 2012 134, 6819-6825.

[94] P. Hoyer, H. Weller, J. Phys. Chem. 1995, 99, 1409614100 .

[95] M. Shim, C. Wang, P. Guyot-Sionnest, J. Phys. Chem. B 2001, 105, 2369-2373.

[96] A. L. Roest, J. J. Kelly, D. Vanmaekelbergh, E. A. Meulenkamp, Phys. Rev. Lett. 2002, 89, 036801-4.

[97] P. Yu, D.; Wang, C; Guyot-Sionnest, Science 2003, 300, 1277-1280.

[98] P. Guyot-Sionnest, C. Wang, J. Phys. Chem. B 2003, 107, 7355-7359.

[99] V. Pinchetti, M. Lorenzon, H. McDaniel, R. Lorenzi, F. Meinardi, V. I. Klimov, S. Brovelli, Nano Lett. 2017, 17, 4508-4517.

[100] B. Huang, H. Yang, L. Zhang, Y. Yuan, Y. Cui, J. Zhang, Nanoscale 2018, 10, 18331-18340.

[101] T. J. Jacobsson, T. Edvinsson, J. Phys. Chem. C 2013 117, 5497-5504.

[102] M. Lu, B. Li, Y. Zhang, W. Liu, Y. Yang, Y. Wang, Q. Yang, Chem. Phys. Lett. 2017, 675, 81-84.

[103] S. C. Boehme, H. Wang, L. D. A. Siebbeles, D. Vanmaekelbergh, A. J. Houtepen, ACS Nano 2013, 7, 2500-2508.

[104] D. Spittel, J. Poppe, C. Meerbach, C. Ziegler, S. G. Hickey, A. Eychmüller, ACS Nano 2017, 11, 12174-12184.

[105] K. S. Jeong, Z. Deng, S. Keuleyan, H. Liu, P. GuyotSionnest, J. Phys. Chem. Lett. 2014, 5, 1139-1143.

[106] M. H. Hudson, M. Chen, V. Kamysbayev, E. M. Janke, X. Lan, G. Allan, C. Delerue, B. Lee, P. Guyot-Sionnest, D. V Talapin, ACS Nano 2018, 12, 9397-9404.

[107] M. Park, D. Choi, Y. Choi, H. Shin, K. S. Jeong, ACS Photonics 2018, 5, 1907-1911.

[108] S. G. Hickey, D. J. Riley, Electrochim. Acta 2000, 45, 3277-3282.

[109] G. M. Carroll, C. K. Brozek, K. H. Hartstein, E. Y. Tsui, D. R. Gamelin, J. Am. Chem. Soc. 2016, 138, 43104313.

[110] K. H. Hartstein, C. K. Brozek, S. O. M. Hinterding, D. R. Gamelin, J. Am. Chem. Soc. 2018, 140, 3434-3442.

[111] G. M. Carroll, E. Y. Tsui, C. K. Brozek, D. R. Gamelin, Chem. Mater. 2016, 28, 7912-7918.

[112] E. Y. Tsui, G. M. Carroll, B. Miller, A. Marchioro, D. R. Gamelin, Chem. Mater. 2017, 29, 3754-3762.

[113] S. Gudjonsdottir, W. van der Stam, N. Kirkwood, W. H. Evers, A. J. Houtepen, J. Am. Chem. Soc. 2018, 140 , 6582-6590.

[114] A. S. Fuhr, H. J. Yun, N. S. Makarov, H. Li, H McDaniel, V. I. Klimov, ACS Photonics 2017, 4, 24252435.

[115] H. Lu, G. M. Carroll, X. Chen, D. K. Amarasinghe, N. R. Neale, E. M. Miller, P. C. Sercel, F. A. Rabuffetti, A. L.
Efros, M. C. Beard, J. Am. Chem. Soc. 2018, 140, 1375313763.

[116] S. C. Boehme, T. A. Walvis, I. Infante, F. C. Grozema, D. Vanmaekelbergh, L. D. A. Siebbeles, A. J. Houtepen, ACS Nano 2014, 8, 7067-7077.

[117] S. C. Boehme, J. M. Azpiroz, Y. V. Aulin, F. C. Grozema, D. Vanmaekelbergh, L. D. A. Siebbeles, I. Infante, A. J. Houtepen, Nano Lett. 2015, 15, 3056-3066.

[118] S. Brovelli, C. Galland, R. Viswanatha, V. I. Klimov, Nano Lett. 2012, 12, 4372-4379.

[119] S. C. Boehme, D. Vanmaekelbergh, W. H. Evers, L. D. A. Siebbeles, A. J. Houtepen, J. Phys. Chem. C 2016, 120, 5164-5173.

[120] S. Brovelli, W. K. Bae, F. Meinardi, B. Santiago González, M. Lorenzon, C. Galland, V. I. Klimov, Nano Lett. 2014, 14, 3855-3863.

[121] M. Lorenzon, S. Christodoulou, G. Vaccaro, J. Pedrini, F. Meinardi, I. Moreels, S. Brovelli, Nat. Commun. 2015, 6, 6434-6442.

[122] L. Jin, Y. Fang, D. Wen, L. Wang, E. Wang, S. Dong, ACS Nano 2011, 5, 5249-5253.

[123] L.-X. Qin, W. Ma, D.-W. Li, Y. Li, X. Chen, H.-B. Kraatz, T. D. James, Y.-T. Long, Chem. - A Eur. J. 2011, 17, 5262-5271.

[124] H. Zhang, Y. Yu, L. Zhang, Y. Zhai, S. Dong, Chem. Sci. 2016, 7, 6721-6727.

[125] Y. Zhai, H. Zhang, L. Zhang, S. Dong, Nanoscale 2016, 8, 9493-9497.

[126] H. Zhang, Y. Zhai, M. Wang, S. Dong, Y. Fang, L. Zhang, Nanoscale 2019, 11, 3718-3724.

[127] Q. Ma, H. Zhang, J. Chen, S. Dong, Y. Fang, Chem. Commun. 2019, 55, 644-647.

[128] C. Kong, D.-W. Li, Y. Li, R. Partovi-Nia, T. D. James, Y.-T. Long, H. Tian, Analyst 2012, 137, 1094-1096.

[129] A. André, M. Weber, K. M. Wurst, S. Maiti, F. Schreiber, M. Scheele, ACS Appl. Mater. Interfaces 2018, 10, 24708-24714.

[130] T. Wang, J. Bai, X. Jiang, G. U. Nienhaus, ACS Nano 2012, 6, 1251-1259.

[131] R. Pokrop, K. Pamuła, S. Deja-Drogomirecka, M. Zagorska, P. Reiss, G. Louarn, F. Chandezon, A. Pron, Mater. Chem. Phys. 2010, 123, 756-760.

[132] C. Querner, P. Reiss, S. Sadki, M. Zagorska, A. Pron, Phys. Chem. Chem. Phys. 2005, 7, 3204-3209.

[133] V. M. Dzhagan, I. Lokteva, C. Himcinschi, J. KolnyOlesiak, M. Y. Valakh, S. Schulze, D. R. T. Zahn, J. Appl. Phys. 2011, 109, 084334.

[134] B. P. Falcão, J. P. Leitão, H. Águas, R. N. Pereira, Phys. Rev. B 2018, 98, 195406-6.

[135] A. G. Rolo, M. I. Vasilevskiy, J. Raman Spectrosc. 2007, 38, 618-633.

[136] V. Dzhagan, I. Lokteva, C. Himcinschi, X. Jin, J. Kolny-Olesiak, D. R. Zahn, Nanoscale Res. Lett. 2011, 6, 79-88.

[137] C. Lin, D. F. Kelley, M. Rico, A. M. Kelley, ACS Nano 2014, 8, 3928-3938.

[138] J. R. Lombardi, R. L. Birke, J. Phys. Chem. C 2014, 118, 11120-11130.

[139] Z. Zeng, W. Zhang, D. M. Arvapalli, B. Bloom, A. Sheardy, T. Mabe, Y. Liu, Z. Ji, H. Chevva, D. H. Waldeck, J. Wei, Phys. Chem. Chem. Phys. 2017, 19, 20101-20109.

[140] Z. Ji, E. Dervishi, S. K. Doorn, M. Sykora, J. Phys. Chem. Lett. 2019, 10, 953-959.

[141] Z. Liang, M. Kang, G. F. Payne, X. Wang, R. Sun, ACS Appl. Mater. Interfaces 2016, 8, 17478-17488.

[142] M. Mattei, G. Kang, G. Goubert, D. V. Chulhai, G. C. Schatz, L. Jensen, R. P. Van Duyne, Nano Lett. 2017, 17, 590-596.

[143] N. Martín Sabanés, T. Ohto, D. Andrienko, Y. Nagata, K. F. Domke, Angew. Chemie - Int. Ed. 2017, 56, 97969801.

[144] J. Clausmeyer, M. Nebel, S. Grützke, Y. U. Kayran, W. Schuhmann, Chempluschem 2018, 83, 414-417.

[145] D. Ibañez, J. Garoz-Ruiz, A. Heras, A. Colina, Anal. Chem. 2016, 88, 8210-8217. 
Received: ((will be filled in by the editorial staff))

Accepted: ((will be filled in by the editorial staff))

Published online: ((will be filled in by the editorial staff)) 
